\title{
Phase transitions of cellulose nanocrystal suspensions from nonlinear oscillatory shear
}

\author{
Sylwia Wojno • Mina Fazilati • Tiina Nypelö • \\ Gunnar Westman $\cdot$ Roland Kádár ${ }^{\mathbb{D}}$
}

Received: 13 August 2021 / Accepted: 6 February 2022 / Published online: 25 February 2022

(C) The Author(s) 2022

\begin{abstract}
Cellulose nanocrystals (CNCs) selfassemble in water suspensions into liquid crystalline assemblies. Here, we elucidate the microstructural changes associated with nonlinear deformations in (2-9 wt\%) CNC suspensions through nonlinear rheological analysis, that was performed in parallel with coupled rheology-polarized light imaging. We show that nonlinear material parameters from Fourier-transform rheology and stress decomposition are sensitive to all CNC phases investigated, i.e. isotropic, biphasic and liquid crystalline. This is in contrast to steady shear and linear viscoelastic dynamic moduli where the three-region behavior and weak strain overshoot cannot distinguish between biphasic and liquid crystalline phases. Thus, the inter-cycle
\end{abstract}

Supplementary Information The online version contains supplementary material available at https://doi. org/10.1007/s10570-022-04474-0.

S. Wojno · M. Fazilati · R. Kádár

Division of Engineering Materials, Department of Industrial and Materials Science, Chalmers University of Technology, 41296 Gothenburg, Sweden

S. Wojno · T. Nypelö · G. Westman · R. Kádár $(\square)$ Wallenberg Wood Science Centre (WWSC), Chalmers University of Technology, 41296 Gothenburg, Sweden e-mail: roland.kadar@chalmers.se

M. Fazilati · T. Nypelö

Division of Applied Chemistry, Department of Chemistry and Chemical Engineering, Chalmers University

of Technology, 41296 Gothenburg, Sweden and intra-cycle nonlinear parameters investigated are a more sensitive approach to relate rheological measurements to $\mathrm{CNC}$ phase behavior.

Keywords Cellulose nanocrystals (CNCs) . CNC water suspensions · Self-assembly phases · Rheology $\cdot$ Birefringence $\cdot$ Fourier-transform rheology $\cdot$ Stress decomposition

\section{Introduction}

Cellulose nanocrystals (CNCs) show enormous potential for many applications, either as a dominant material component or part of multicomponent systems. CNCs are rod-like nanoparticles which are crystalline having a long axis with a slight twist (Conley et al. 2017). Their dimensions depend on the cellulose

\author{
Present Address: \\ M. Fazilati \\ Faculty of Health and Society, Biofilms Research Center \\ for Biointerfaces, Malmö University, 20506 Malmö, \\ Sweden \\ G. Westman \\ Division of Chemistry and Biochemistry, Department \\ of Chemistry and Chemical Engineering, Chalmers \\ University of Technology, 41296 Gothenburg, Sweden
}


source and extraction method (Dong et al. 1998) and are usually $4-10 \mathrm{~nm}$ in width and $100-1000 \mathrm{~nm}$ in length (Habibi et al. 2010; Klemm et al. 2011; Navarro et al. 2021). Their crystalline nature is the result of removing amorphous segments from the cellulose source of choice. In addition, using sulfuric acid for the extraction results in CNC particles with sulfate half-ester groups on their surface. This leads to electrostatically stabilized CNC aqueous suspensions (Marchessault et al. 1959; Revol et al. 1992; Dong et al. 1996). In static conditions (no flow), dilute aqueous suspensions of $\mathrm{CNCs}$ form an isotropic phase. As the CNC concentration is increased above a critical concentration, the suspensions assemble into a biphasic system consisting of isotropic and liquid crystalline ordered domains. The formation of ordered domains with increasing concentration has been described theoretically by Onsager (1949) who elaborated how suspensions of nanorods can spontaneously develop long-range orientational order as a result of the gain in translational entropy overcompensating the loss in rotational entropy. An additional increase in concentration will lead to a complete liquid crystalline phase (Kádár et al. 2021). The liquid crystalline (LC) domains comprise chiral nematic and/or nematic CNC structures (Revol et al. 1992; Abitbol and Cranston 2014). Chiral nematic crystals are characterized by a cholesteric pitch, a distance along the helical axis that separates nanorods of the same orientation after a $360^{\circ}$ rotation (Habibi et al. 2010). In polarized light microscopy, chiral nematic orders in biphasic and liquid crystalline suspensions can be observed in the form of a 'fingerprint pattern', where the lines indicate the pitch of the chiral order (Revol et al. 1992). The nematic phase refers to a crystal orientational order having the $\mathrm{CNCs}^{\text {' }}$ long axes approximately parallel, for distances significantly higher than the dimensions of the nanorods (Dufresne 2012). Further increasing the CNC concentration results into a (repulsive) glassy phase (Xu et al. 2018). Their hierarchical structure makes CNC suspensions, and films resulting therefrom, optically active materials which display birefringence. Birefringence can arise from any repeating pattern in a structure, chiral nematic and nematic being two of them (Ureña-Benavides et al. 2011; Dumanli et al. 2014). Subjecting CNC suspensions to flow can cause preferential orientation in the flow direction (Hubbe et al. 2017). The birefringence properties of CNC can be used in films as optical filters for color filters, smart clothes, and in solar-gain-regulating building technologies, free-standing iridescent photonic films with tunable structural colors to detect changes in the environment or in decorative coatings (Haniffa et al. 2016; Fernandes et al. 2017). The self-assembly of CNCs is known to depend on many factors, such as surface charge, ionic strength, aspect ratio, concentration, sonication, temperature, etc. (Dong et al. 1998; Shafeiei-Sabet et al. 2013; Abitbol et al. 2018; Derakhshandeh et al. 2013; Zhang et al. 2018).

Nonlinear rheological analysis, such as FourierTransform (FT) Rheology and stress decomposition, can increase measurement sensitivity and have the potential to reveal material response features that are not otherwise observable in linear viscoelastic measurements (Wilhelm and van Dusschoten 2001; Hyun et al. 2011). The nonlinear oscillatory shear analysis has gained increasing attention as a rheological testing method especially in polymer melts and solutions, filled polymeric systems, suspensions and emulsions (Hyun et al. 2011). However, the nonlinear viscoelastic rheological characterization of CNC suspensions is not well established. Chen et al. (Chen et al. 2017) investigated the behavior of CNC particles in PVA and CMC aqueous solutions with respect to linear viscoelastic dynamic moduli in the nonlinear region. They reported that the PVA has stronger interactions with negatively charged CNC particles, resulting in a lower percolation threshold of $\mathrm{CNC}$ in the PVA solution during Small Amplitude Oscillatory Shear (SAOS) and a weak strain overshoot behavior in Large Amplitude Oscillatory Shear (LAOS). However, we note that linear viscoelastic dynamic moduli do not accurately represent nonlinear material response and that nonlinear material parameters were not further evaluated. Van den Berg et al. (2018) studied the interfacial layers of CNC particles in (interfacial) SAOS, LAOS and large amplitude oscillatory dilatation (LAOD). Lissajous-Bowditch diagrams in LAOS exhibited strain stiffening, yielding, and flow of the interfacial layers for the high degree of chemical substitution. Another study on CNC in aqueous media with added $\mathrm{NaCl}$ revealed that the nonlinear viscoelastic measurements are significantly sensitive to detect changes in the microstructure and yield valuable information about the network structure (Abbasi Moud et al. 2020). Furthermore, performing the nonlinear oscillatory shear analysis 
enabled detecting the formation of a double network in CNC-PVA/salt hydrogels at high CNC loadings (Moud et al. 2021). Several studies indicate that some materials can exhibit unique nonlinear signatures with a non-quadratic scaling of $I_{3 / 1}$ with the strain amplitude, $I_{3 / 1} \propto \gamma_{0}^{n}, n \neq 2$, that could be related to microstructural or molecular material properties. Hyun et al. (2007) reported a lower slope of $I_{3 / 1}$ for long-branched polymers compared to linear polymers in the MAOS region, which could be used as a measure of the degree of branching. There has been evidence that percolated nanocomposite polymer systems can exhibit peculiar nonlinear scaling behavior (Kádár et al. 2017; Gaska and Kádár 2019). Natalia et al. (2020) reported all evidence to date of noninteger power-law expansions for nonlinear viscoelastic properties. Building on previous evidence, Kádár et al. (2020b) determined that the onset of unique nonlinear signatures, dubbed 'nonlinear oddities', in the MAOS region matches the electrical percolation threshold of hierarchical reduced graphene oxide (HrGO)-polypropylene (PP) nanocomposites and subsequent percolated network consolidation. Meurer et al. (2021) applied the same analysis framework for chemical gels in the form of pressure sensitive adhesives. Changes in nonlinear signatures were recorded around the gel-point, however, both inter-cycle and intra-cycle nonlinear behavior differed considerably from those recorded in polymer nanocomposites. Associating such 'nonlinear oddities' to physical gels in the form of suspensions has yet to be elucidated.

Optical visualization techniques combined with rheological characterization can help understand the relationship between microstructure, phase transitions, and rheological properties (Kádár et al. 2021). One of the commonly used rheological hyphenated methods for optically active suspensions is polarized light imaging (PLI). Shafiei-Sabet et al. (2012) investigated the effect of temperature and ultrasound energy using the rheo-PLI technique. These two factors affect the suspensions' microstructure depending on the concentration and the pitch of the chiral nematic domains. The degree of sulfation of $\mathrm{CNC}$ particles also significantly affects the phase transitions from isotropic through the liquid crystal, to a gel appearance (Shafeiei-Sabet et al. 2013). Hausmann et al. (2018) studied the dynamics of CNC alignment with high weight fractions (10-40 wt\%) of particles under shear flow coupled with PLI. Kádár et al. (2020a) reported microphase transition sequences of CNC suspensions in shear flow through combined rheology and polarized light optical visualizations. Fazilati et al. (2021) combined rheology with polarized light imaging to elucidate the thixotropic behavior of CNCs suspended in water.

Although the CNC particles were first described in 1949 by Rånby et al. (1949), and numerous factors affecting crystalline phases in suspension identified, there is still a gap in a clear understanding of phase transitions and their relationship to the rheological behavior of CNC suspensions. It is common to use steady shear and (linear viscoelastic) oscillatory shear measurements to associate rheological behavior to CNC phases, however, a phase identification therewith remains a challenge. Our hypothesis is that nonlinear oscillatory shear analysis through increased measurement sensitivity and additional nonlinear rheological parameters is a more sensitive framework to elucidate CNC phases from rheology. To our knowledge, this is the first study to address this. To provide additional insight into the connection between the nonlinear material response of the suspensions and microstructural developments in the hierarchy of the suspensions we analyze the combined rheo-PLI optical visualizations in oscillatory strain sweep tests. While rheological measurements capture the bulk material response corresponding to all levels of the CNC hierarchy, macroscopic PLI data captures only the higher levels of the hierarchy, i.e. self-assembled tactoids/nematic/chiral nematic CNCs distributed in a polydomain structure within the measurement gap, and their orientation dynamics (Fazilati et al. 2021).

\section{Experimental section}

\section{Materials}

Cellulose nanocrystals (CNCs) in powder form were purchased from CelluForce, CELLUFORCE NCV100 - NASD90 (Montreal, Canada), having sulfur content of about $0.9 \%$ and sulfate content of about $250 \mu \mathrm{mol} / \mathrm{g}$. According to X-ray photoelectron spectroscopy (XPS) analysis sulfur content was 0.39 \pm 0.04 atom\% that was performed using a PHI 5000 VersaProbe III Scanning XPS Microprobe at an angle of $45^{\circ}$. Aqueous $\mathrm{CNC}$ suspensions in the presence of $0.1 \mathrm{mM} \mathrm{NaCl}$ salt showed an average zeta potential 
value $-32.0 \pm 3.4 \mathrm{mV}$, see Table $\mathrm{S} 1$ in the Supplementary information. Suspensions of $2-9 \mathrm{wt} \% \mathrm{CNC}$ concentrations were prepared with deionized water (Millipore Milli-Q Purification System) and stirred on a bench shaker for $24 \mathrm{~h}$. Prior to all rheological measurements, each suspension was treated for 15 min in an ultrasound bath (Bransonic Ultrasonic Bath 221, Connecticut, U.S.) operating at $50 \mathrm{kHz}$ and 235 W.

$\mathrm{CNCs}$ are reported with length between 100 and $300 \mathrm{~nm}$. The diameter is typically between 4 and $10 \mathrm{~nm}$ (Qing et al. 2013; Saito et al. 2009). We have determined the diameter of the CNCs to be 4.1 $\pm 1.0 \mathrm{~nm}$ (Navarro et al. 2021; Fazilati et al. 2021) and hence deem the aspect ratios of the CNCs to be between 25 and 75 .

\section{Optical microscopy}

Transmission optical microscopy was carried out with a Carl Zeiss A1 (Oberkochen, Germany) optical microscope equipped with linear polarizers in a cross-polarizer setup. Each sample was placed and squeezed in the space between two microscope slides after 15 min sonication in an ultrasound bath.

\section{Rheological characterization}

All rheological measurements were performed on an Anton Paar MCR 702 TwinDrive rheometer (Graz, Austria) at $23{ }^{\circ} \mathrm{C}$. Before measurement, each sample was allowed to relax for $240 \mathrm{~s}$ after moving to the gap position (1 $\mathrm{mm}$ for all tests). Two sets of configurations were used: (i) a single motor-transducer setup for rheo-PLI and (ii) a separate motor-transducer setup for nonlinear oscillatory shear analysis.

\section{Rheo-PLI}

Rheo-PLI experiments were performed in a single motor-transducer configuration using a custom rheooptical visualization setup based on the P-PTD200/ GL accessory with a parallel-plate geometry of $(2 R=) 43 \mathrm{~mm}$ diameter. Two linear polarizers were placed at $45^{\circ}$ relative orientation above and below the parallel-plate setup, respectively. HD video recordings $(1280 \times 720 \mathrm{px})$ were performed at $60 \mathrm{fps}$ from below, perpendicularly to the shear plane, using a Canon DSLR camera setup comprising a Canon
L-series $100 \mathrm{~mm}$ macro lens combined with a Canon $25 \mathrm{~mm}$ extension tube (Tokyo, Japan). More details about the setup can be found elsewhere (Kádár et al. 2020a), while a schematic diagram of the setup can be found in Fig. S1 (Supplementary information). Space-time diagrams were constructed from the video recordings by extracting one line of pixels at a fixed position out of each still frame and appended to a new image having the $\mathrm{x}$-axis corresponding to the experimental time and the $y$-axis corresponding to the length $L$ (Kádár et al. 2020a), see also Fig. S1. Both oscillatory and steady shear measurements were performed on the setup. The strain sweep measurements were performed at 0.6 and $4 \mathrm{rad} / \mathrm{s}$ with the strain amplitude ranging from 0.01 to $1500 \%$. In turn, the steady shear measurements were conducted within the shear rate range of 0.001 to $100 \mathrm{~s}^{-1}$ using a custom procedure for steady-state detection (Kádár et al. 2020a).

\section{Nonlinear oscillatory shear analysis}

In the separate motor-transducer configuration, linear and nonlinear oscillatory shear measurements were performed using a parallel-plate geometry of $(2 R=) 50 \mathrm{~mm}$ in diameter. Strain sweep measurements were performed within a strain amplitude range of 0.01 to $1500 \%$ at the following frequencies: $0.6,1,2$, and $4 \mathrm{rad} / \mathrm{s}$. Performing measurements at different angular frequencies could reveal essential information about the microstructure, e.g. through the dependency of $I_{3 / 1}$ on $\omega$ (Kádár et al. 2020b). The nonlinear data analysis of the shear stress output signal was performed in the framework of Fourier-Transform Rheology and stress decomposition analysis. A graphical overview thereof can be found in Fig. 1. One simple way to distinguish between linear and nonlinear oscillatory shear is to consider the measured time dependent shear stress response and its corresponding Fourier spectra. In linear viscoelastic oscillatory shear, a sinusoidal shear strain input $\gamma(t)=\gamma_{0} \sin (\omega t)$, where $\gamma_{0}$ is the strain amplitude and $\omega$ the angular frequency, will result in a sinusoidal shear stress output $\sigma(t)=\sigma_{0} \sin (\omega t+\delta)$, shifted with the phase angle $\delta$. Accordingly, the Fourier spectrum of the shear stress contains only the fundamental intensity $I_{1}$ of the imposed $\omega$, see Fig. 1a. In contrast, for nonlinear deformations a sinusoidal strain input translates into a distorted shear stress output which 


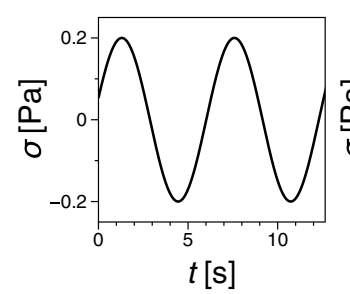

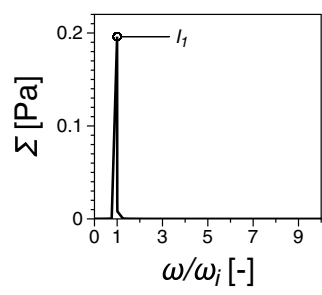

(a)
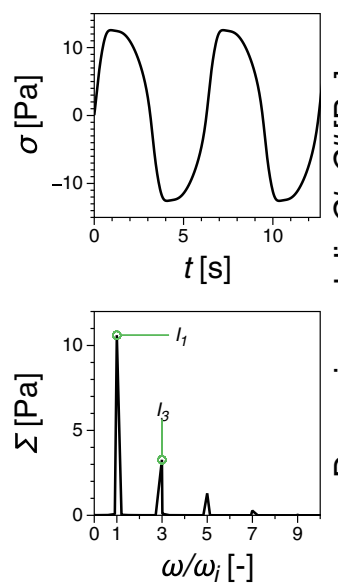

(b)

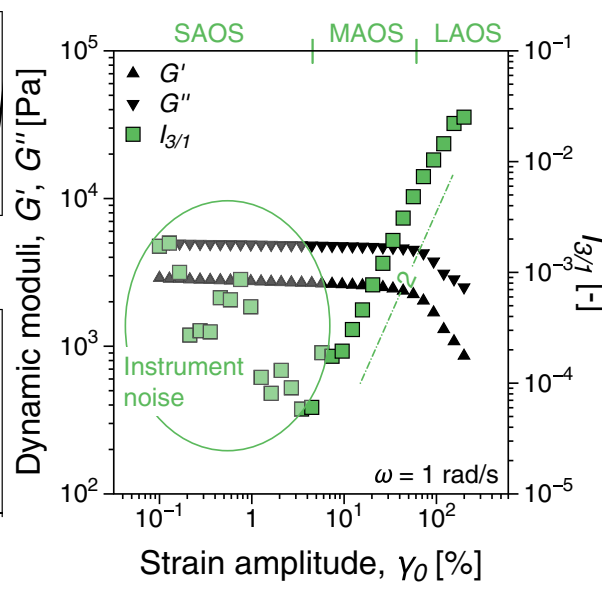

(c)
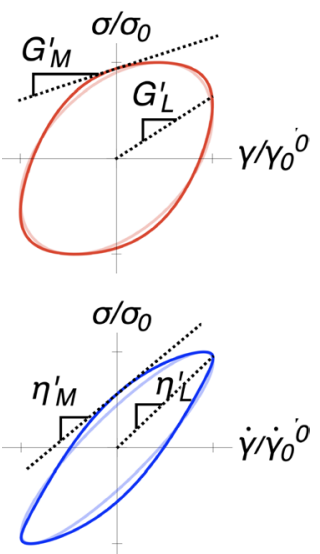

(d)
Fig. 1 Generic graphical overview of the nonlinear analysis: a linear material response as expressed by the sinusoidal variation of the measured shear stress, $\sigma$, in the time domain and the corresponding Fourier spectra; b nonlinear material response as expressed by the non-sinusoidal variation of the measured shear stress in the time domain and the corresponding Fourier spectra; c generic example comparing the linear

leads to higher harmonics in the corresponding Fourier spectra, in addition to the fundamental intensity, $I_{n}, n=3,5 \ldots$, (Hyun et al. 2011) see Fig. 1b. This can also be translated into the behavior of the linear viscoelastic dynamic moduli, the storage and loss modulus $G^{\prime}, G^{\prime \prime}$, in dynamic strain sweep tests, which is how the linear viscoelastic limit is usually quantified. For strain amplitudes where the shear stress output is sinusoidal the elastic and loss modulus are independent of the strain amplitude. However, as the applied strain amplitude is increased and the shear stress output becomes distorted, the storage and loss moduli become functions of the applied strain amplitude i.e. $G^{\prime}=G^{\prime}\left(\gamma_{0}\right), G^{\prime \prime}=G^{\prime \prime}\left(\gamma_{0}\right)$, see Fig. 1c. Thus, it should be noted that in this case the linear viscoelastic dynamic moduli contain an unreported intrinsic error owing to the distorted shear stress signals. This nonlinear component of the material response can be quantified using nonlinear material parameters. The third relative higher harmonic, $I_{3 / 1} \equiv I_{3} / I_{1}$, can be used to characterize the shear stress signal nonlinearities, as $I_{3}$ contains the largest nonlinear contribution to the Fourier spectra (Hyun et al. 2011). For small strain amplitudes (small amplitude oscillatory shear, SAOS) $I_{3 / 1}$ is scattered with general scaling behavior viscoelastic dynamic moduli, $G^{\prime}, G^{\prime \prime}$, and the third relative higher harmonic, $I_{3 / 1}=I_{3} / I_{1}$, from Fourier-transform analysis; d elastic (top) and viscous (bottom) Lissajous-Bowditch (LB) diagrams illustrating the tangent slopes at zero strain/rate $\left({ }_{M}\right)$ and secants at maximum strain/rate $\left({ }_{L}\right)$. The light red and blue represent elliptic LB diagrams corresponding to a linear viscoelastic response, respectively

of $I_{3 / 1} \propto \gamma_{0}^{-1}$ corresponding to nonlinearities due to instrumentation noise (Hyun et al. 2011). In this framework, the onset of the nonlinear behavior is marked by a steep increase in $I_{3 / 1}$. It can be shown through theoretical reasoning (Hyun and Kim 2011) that $I_{3 / 1} \propto \gamma_{0}^{2}$ and this strain amplitude region is called medium amplitude oscillatory shear (MAOS). For even higher strain amplitudes, i.e. large amplitude oscillatory shear (LAOS), the quadratic scaling is lost (Fig. 1c). In addition to quantifying directly the nonlinearity of the shear stress signal, we briefly note that by taking as example the data presented in Fig. 1c the dynamic range of $I_{3 / 1}$ spans approximately 3 decades compared to less than one decade in $G^{\prime}, G^{\prime \prime}$.

In addition to material parameters based on shear stress Fourier spectra, which essentially quantify how distorted the shear stress signal is, nonlinear material parameters can also be derived based directly on the intra-cycle distortion of the shear stress. The elastic $\left(\sigma(t) / \sigma_{0}\right.$ vs. $\left.\gamma(t) / \gamma_{0}\right)$ and viscous $\left(\sigma(t) / \sigma_{0}\right.$ vs. $\left.\dot{\gamma}(t) / \dot{\gamma}_{0}\right)$ Lissajous-Bowditch (LB) diagrams can therefore be used to assess the nonlinear material response (Ewoldt et al. 2008). An elliptic shaped response indicates a linear viscoelastic behavior, see the light red and light blue 
loops in Fig. 1c. Concurrently, any deviations due to distorted shear stress signals are characteristic of nonlinear viscoelastic material response (non-elliptic) signatures, see Fig. 1c. Ewoldt et al. (2008) defined two elastic moduli and two dynamic viscosities, see also Fig. 1c,

$\left.G_{M}^{\prime} \equiv \frac{d \sigma}{d \gamma}\right|_{\gamma=0},\left.G_{L}^{\prime} \equiv \frac{\sigma}{\gamma}\right|_{\gamma=\gamma_{0}}$

where $G_{M}^{\prime}$ is the minimum-strain and $G_{L}^{\prime}$ is the largestrain modulus, and

$\left.\eta_{M}^{\prime} \equiv \frac{d \sigma}{d \dot{\gamma}}\right|_{\dot{\gamma}=0},\left.\eta_{L}^{\prime} \equiv \frac{\sigma}{\dot{\gamma}}\right|_{\dot{\gamma}=\gamma_{0}}$

where $\eta_{M}^{\prime}$ is the minimum-rate and $\eta_{L}^{\prime}$ the large-rate viscosity, to quantify the intra-cycle material behavior based on LB diagrams. These parameters were used to define the strain-stiffening $(S)$ and shear-thickening (T) dimensionless ratios (Ewoldt et al. 2008):

$S \equiv \frac{G_{L}^{\prime}-G_{M}^{\prime}}{G_{L}^{\prime}}$

$T \equiv \frac{\eta_{L}^{\prime}-\eta_{M}^{\prime}}{\eta_{L}^{\prime}}$

where $S>0$ indicates intra-cycle strain-stiffening, $S<0$ intra-cycle strain-softening, $T>0$ intra-cycle shear-thickening and $T<0$ intra-cycle shear-thinning.

\section{Results and discussion}

Morphological characterization

Polarized optical microscopy (POM) was used to capture micrographs of the suspensions investigated to observe CNC phases independently from the rheological characterization, (Fig. 2). No birefringence is observed for $2 \mathrm{wt} \% \mathrm{CNC}$, which is therefore categorized as isotropic. As concentration increases, the micrographs show birefringence which is deemed to be due to the formation of liquid crystalline domains. The biphasic textures are visible for 3-5 wt $\%$ CNC suspensions, where a liquid crystal fraction coexists as bright droplets with a dark isotropic phase. After reaching the critical concentration from biphasic to liquid crystalline, there is no visible dark isotropic phase and they merge into one domain for 6-9 wt\% CNC. Due to the absence of clear 'fingerprint' textures in the micrographs, we consider the evidence for whether we have nematic or chiral nematic structures in the CNC samples investigated, inconclusive. To summarize, using the given preparation conditions, the $2 \mathrm{wt} \% \mathrm{CNC}$ suspension is isotropic, 3-5 wt $\%$ CNC are biphasic, and 6-9 wt\% CNC are liquid crystalline. We note that biphasic compositions in the range of 3-6 wt\% have been identified for aspect ratios of $61 \pm 40$ (Honorato-Rios et al. 2018).
$2 \mathrm{wt} \% \mathrm{CNC}$

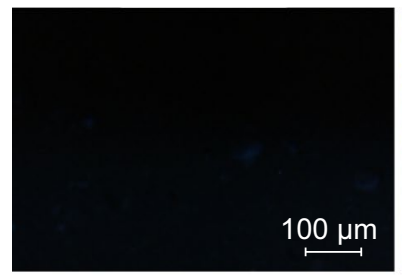

$6 w t \% \mathrm{CNC}$

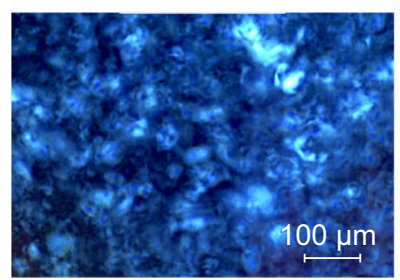

$3 w t \% \mathrm{CNC}$

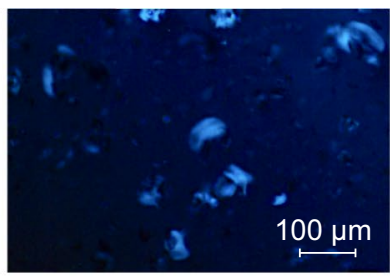

7 wt $\%$ CNC

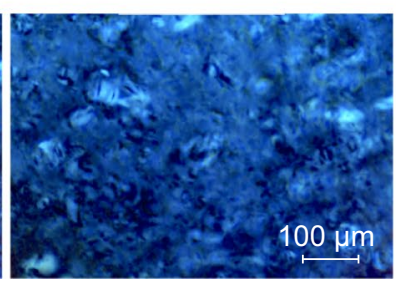

$4 \mathrm{wt} \% \mathrm{CNC}$

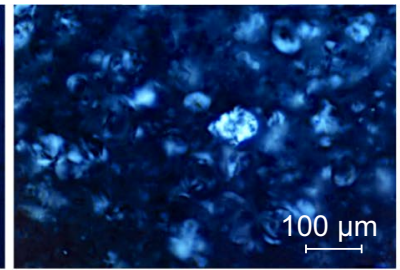

$8 w t \% \mathrm{CNC}$

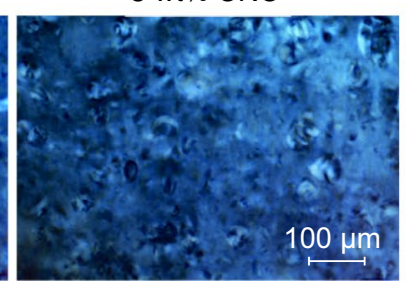

$5 \mathrm{wt} \% \mathrm{CNC}$

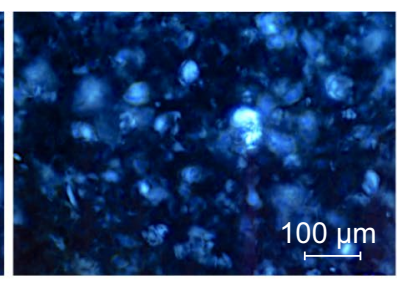

9 wt $\%$ CNC

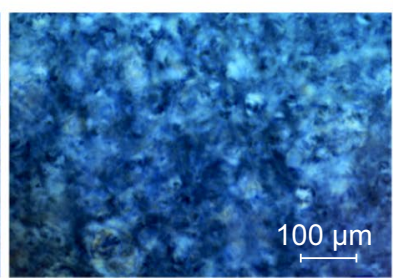

Fig. 2 Polarized optical micrographs for the CNC suspensions studied at rest (no flow) 
Steady and oscillatory shear tests

Here we present steady shear and linear viscoelastic oscillatory shear tests, which are the most common types of tests employed for the characterization of CNC suspensions. The birefringence data from rheoPLI steady shear tests is also discussed to underline the correlation between the birefringent properties of the suspensions under shear and their phase behavior. Rheo-PLI data from the oscillatory shear measurements is discussed after the nonlinear analysis.

The steady shear viscosity functions of the CNC suspensions investigated are shown in Fig. 3. From a qualitative point of view, the steady shear viscosity of the suspensions could be grouped into two categories. In the first category, a zero-shear viscosity Newtonian plateau was apparent followed by a shear-thinning region. Such viscosity functions are characteristic of isotropic phase suspensions, see $2 \mathrm{wt} \% \mathrm{CNC}$. The second group corresponds to 3-9 wt\% $\mathrm{CNC}$, where the suspensions showed evidence of a three-region steady shear viscosity function, which is characteristic of liquid crystal systems (Kádár et al. 2021). Onogi and Asada (1980) were the first to assign the three-region behavior in polymer liquid crystals, however, there is overall quite a large discrepancy among subsequent studies on the estimation of CNC phases based on the three-region viscosity curve. For example, Orts et al. (1998) and Shafiei-Sabet et al. (2012), Shafeiei-Sabet et al. (2013) assigned the three-region behavior to the liquid crystalline phase. In contrast, Ureña-Benavides et al. (2011) identified this behavior for the biphasic phase. Another example is the

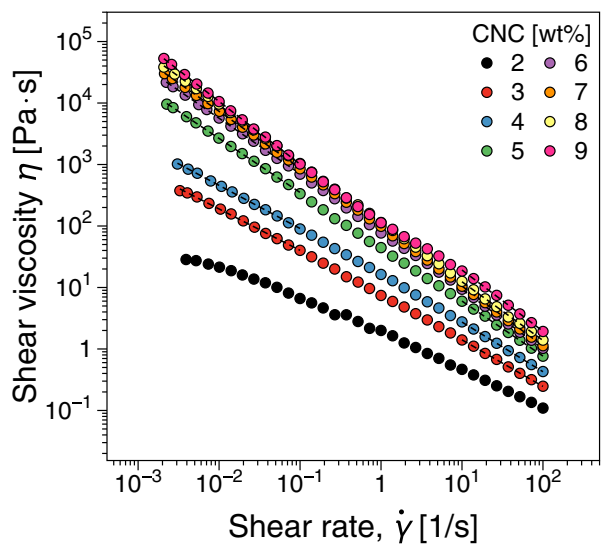

Fig. 3 Steady shear viscosity of the CNC suspensions study of Haywood et al. (2017) where the threeregion behavior was observed in both biphasic and liquid crystalline phases. We note that Haywood et al. (2017) were able to distinguish more than on shear thinning region beyond what could be visually easily assessed by fitting the low and high shear rate regions with the Power-law and comparing the flow indices. Similar observations were made by Fazilati et al. (2021) for suspensions differing only slightly in the preparation steps compared to the present study. In the earliest estimation, Onogi and Asada (1980) hypothesized that at low shear rates in Region I, the polydomain chiral structures are not altered, and domains are able to flow over each other, which results in the first shear-thinning region. At intermediate shear rates, Region II is observed, sometimes called the Newtonian region, where the orientation of the initially unaffected polydomain starts to change. Haywood et al. (2017) identified through Rheo-SANS measurements that the anisotropy in this region remains relatively constant, with an isotropic ring still visible, which could indicate that only part of the rods align in the flow direction, with a significant part of the material remaining randomly oriented. The liquid crystal alignment occurs in Region III, which is a secondary shear-thinning region of the viscosity curve. We also note that for the higher concentrations, above $5 \mathrm{wt} \%$, the relative change in the viscosity is comparatively small. This phenomenon could be explained by considering the Brownian motion of the particles and van der Waals interactions (Dufresne 2012). At a low $\mathrm{CNC}$ concentrations of aqueous suspensions, the rate of particle Brownian rotation is considerable and prevents alignment of the particles (Derakhshandeh et al. 2013). As concentration increases, CNC particles can (locally) orient and form nematic and chiral nematic structures. Similar observations were made by Pignon et al. (2021) who investigated CNC suspensions (2-12.2 wt\%) using two combined techniques, rheo-SAXS and rheo-SALS.

Overall, from the present data, including also previous findings on very similar systems (Kádár et al. 2020a; Fazilati et al. 2021), assigning the three-region behavior to a particular phase remains ambiguous. While power law flow indices, see Eq. S1 in the Supplementary information, of low and high shear rates show a similar behavior with the results of Haywood et al. (2017), see Fig. S2 and Table S2 in the Supplementary information, the three-regions are visually 
apparent only between 5 and 9 wt $\%$ CNC concentrations and even therein not fully consistent, e.g. see 7-8 wt\% in Fig. S2. However, a relatively small change in the viscosity for concentrations 6-9 wt $\%$, compared to lower concentrations, could indicate the suspensions are in the LC phase.

Phase transitions in CNCs can also be inferred based on the birefringence patterns of the suspensions in flow. Fig. 4 presents the evolution of CNC suspensions flow with shear rate as PLI images corresponding to the tests in Fig. 3. The isotropic $2 \mathrm{wt} \% \mathrm{CNC}$ suspension did not indicate changes in color through all applied shear rates. However, at $\dot{\gamma}=23 \mathrm{~s}^{-1}$, a Maltese-cross pattern was observed. This pattern indicates a parallel orientation of one of the main axes of the optical indicatrices of a birefringent material, which is $\mathrm{CNC}$, in the plane of polarization of the incident light (Völker-Pop 2014). As concentration increases, the Maltese-cross appeared at lower shear rates. For the biphasic $3 \mathrm{wt} \% \mathrm{CNC}$, it

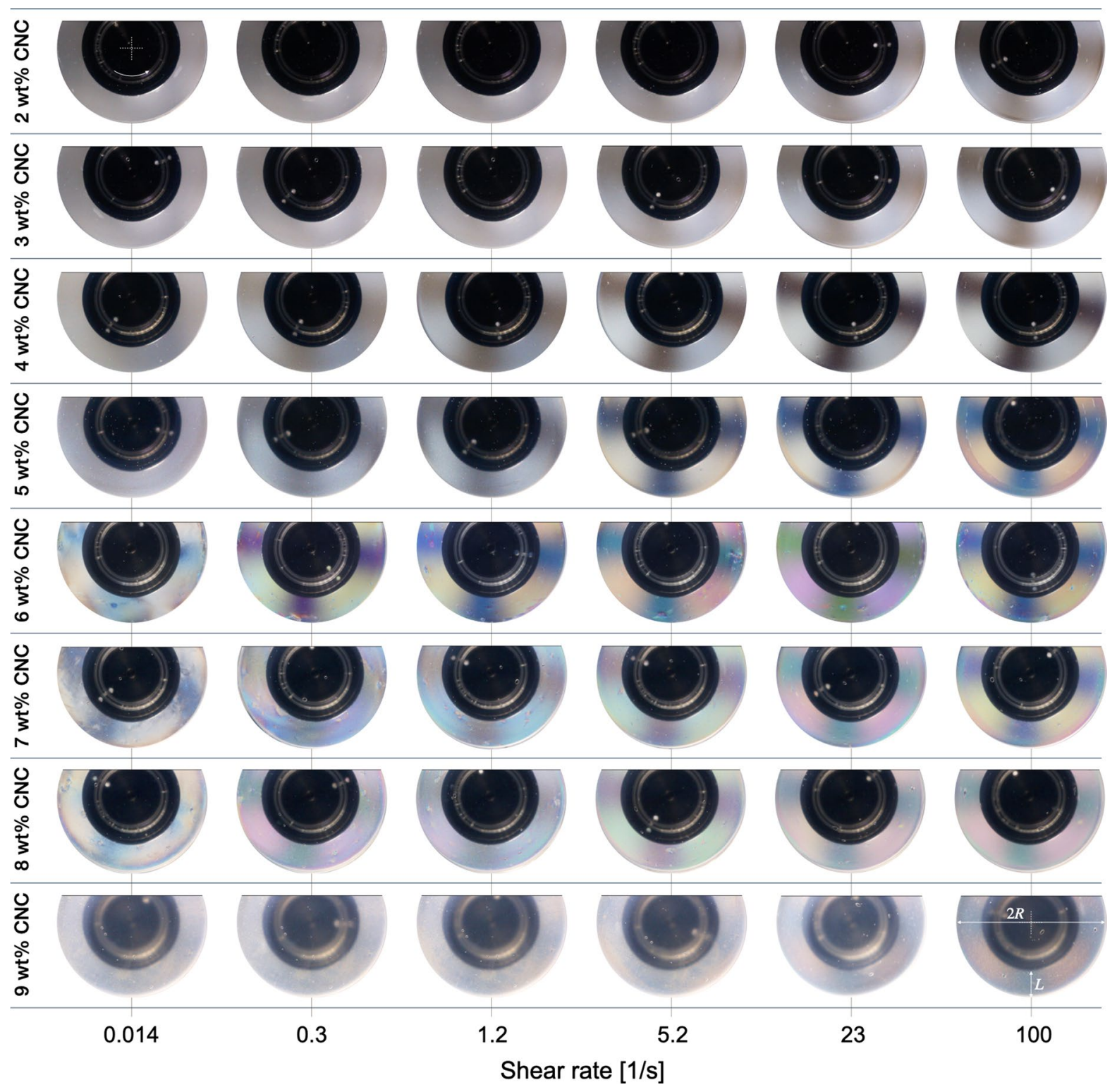

Fig. 4 Still frames showing the birefringence patterns at selected shear rates from the steady shear measurements in Fig. 3 
was observed at $\dot{\gamma}=5 \mathrm{~s}^{-1}$, while for $4 \mathrm{wt} \% \mathrm{CNC}$ at $\dot{\gamma}=0.3 \mathrm{~s}^{-1}$. For concentrated biphasic (5 wt $\% \mathrm{CNC}$ ) and all LC concentrations, a Maltese cross could be observed at $\dot{\gamma}=0.1 \mathrm{~s}^{-1}$. Additionally, as the fraction of the liquid crystal phase increased, a colorful pattern was observed both as large isochromatic areas at the beginning of the tests and in the Maltese-cross patterns. We note that while a color-less suspension prior to the beginning of the tests $(\dot{\gamma}=0)$ followed by a colored Maltese-cross pattern generally signals a biphasic suspension and large isochromatic areas at $\dot{\gamma}=0$ generally suggest liquid crystalline phase suspensions, the observed birefringence patters cannot be used to accurately determine CNC phase transitions with increasing concentration.

Dynamic strain sweep measurements, at two different angular frequencies, 0.6 and $4 \mathrm{rad} / \mathrm{s}$, are presented in Fig. 5. The rheological behavior of isotropic $2 \mathrm{wt} \% \mathrm{CNC}$ was dependent on the applied angular frequency. For $\omega=0.6 \mathrm{rad} / \mathrm{s}$ the suspension exhibited gel-like behavior, $G^{\prime} \cong G^{\prime \prime}$, however, for $\omega=4$ $\mathrm{rad} / \mathrm{s}$, the same concentration exhibited liquid-like behavior, $G^{\prime \prime}>G^{\prime}$. This can indicate that at $2 \mathrm{wt} \%$ $\mathrm{CNC}$ the suspension contains a weak gel-like CNC network. All higher concentrations, 3-9 wt\% CNC, exhibited gel-like behavior, $G^{\prime}>G^{\prime \prime}$, independently of the applied $\omega$. The biphasic 5 wt $\%$ CNC suspension exhibited a weak strain overshoot (WSO) whereby $G^{\prime \prime}$ locally increases at the transition to the nonlinear viscoelastic regime. The WSO is further observed for all higher concentrations, $>5 \mathrm{wt} \% \mathrm{CNC}$. In general, a higher load of CNC particles would lead to stronger associations that can resist restructuring at large deformations causing the overshoot in $G^{\prime \prime}$. We note that while the $\omega$-dependent liquid/gel-like behavior is characteristic solely of the isotropic $2 \mathrm{wt} \%$ $\mathrm{CNC}$, the onset of WSO behavior is well withing the biphasic concentration range and also characterizes the liquid crystalline concentrations.

CNC phase bahavior from nonlinear oscillatory shear analysis

The nonlinear material response as expressed by the third relative higher harmonic, $I_{3 / 1}$, from the FT analysis of dynamic strain sweep measurements is presented in Fig. 6. The instrumentation noise in the SAOS region is represented by semi-transparent datapoints. For the isotropic $2 \mathrm{wt} \% \mathrm{CNC}$ suspension, Fig. 6a, the nonlinearities were detected at the highest recorded strain amplitudes, as expected considering that the suspension viscosity leads to limiting torques for the geometry used (Wojno et al. 2019). A strong $\omega$ dependence in the MAOS region was observed. For $\omega=0.6,1 \mathrm{rad} / \mathrm{s}$ two scaling regions, with $I_{3 / 1} \propto \gamma_{0}^{n}$, where (i) $n \approx 1.8$ for $\gamma_{0} \in[3,10] \%$ and (ii) $n \approx 0.5$ for $\gamma_{0} \in[12,60] \%$ can be distinguished. A decrease in $I_{3 / 1}$ magnitude was recorded with increasing $\omega>1$ $\mathrm{rad} / \mathrm{s}$. For $\omega=2 \mathrm{rad} / \mathrm{s}$ two scaling regions remained identifiable: (i) $n \approx 2$ for $\gamma_{0} \in[6,23] \%$, (ii) $n \approx 0.85$ for $\gamma_{0} \in[24,95] \%$. However, for $\omega=4 \mathrm{rad} / \mathrm{s}$, the behavior approached one scaling region, where $n \approx 1.9$ for $\gamma_{0} \in[15,95] \%$, thus approaching the quadratic scaling theoretically expected. The tendency towards quadratic scaling with increasing $\omega$, for nonlinear signatures where $I_{3 / 1}=I_{3 / 1}(\omega)$, has also been observed in percolated polymer nanocomposites.
Fig. 5 Linear viscoelastic dynamic moduli from strain sweep measurements for $\mathbf{a}$ $\omega=0.6 \mathrm{rad} / \mathrm{s}$ and $\mathbf{b} \omega=4$ $\mathrm{rad} / \mathrm{s}$

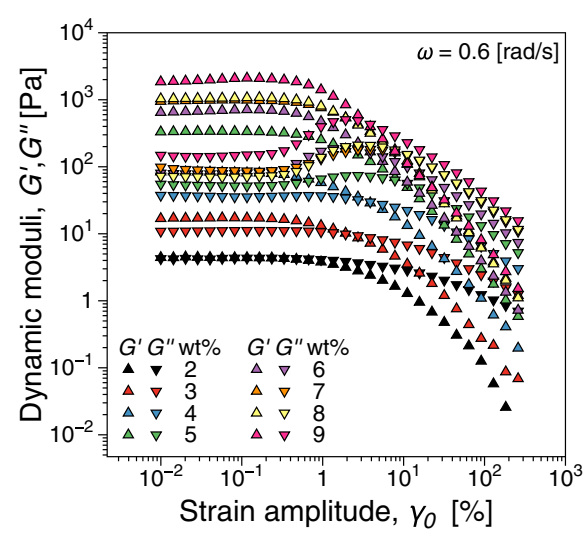

(a)

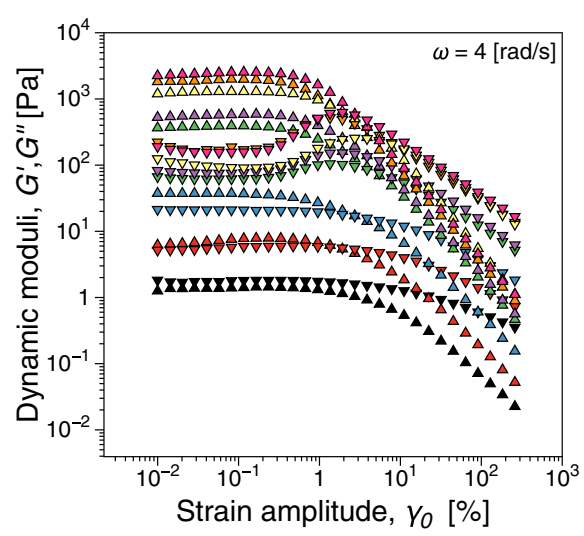

(b) 
Fig. 6 Third relative higher harmonic, $I_{3 / 1}$, from dynamic strain sweeps for $\omega=0.6,1,2,4 \mathrm{rad} / \mathrm{s}: \mathbf{a} 2$ wt $\%$, b 3 wt $\%$, c 4 wt $\%$, d 5 wt $\%$, e 6 wt $\%$, f 7 wt $\%$, g 8 wt $\%$, h 9 wt $\%$ CNC

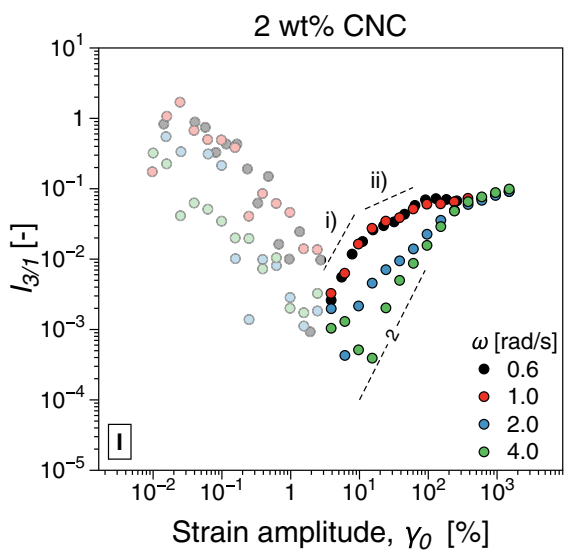

(a)

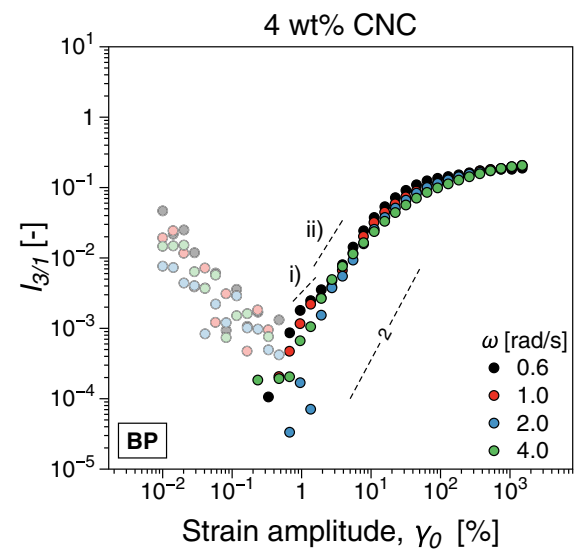

(c)

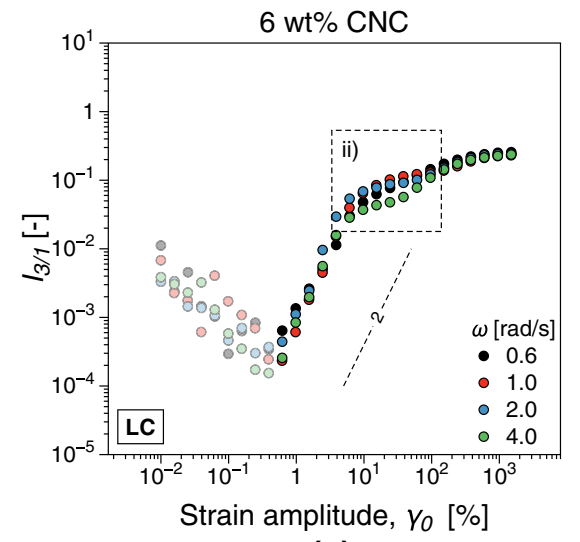

(e)

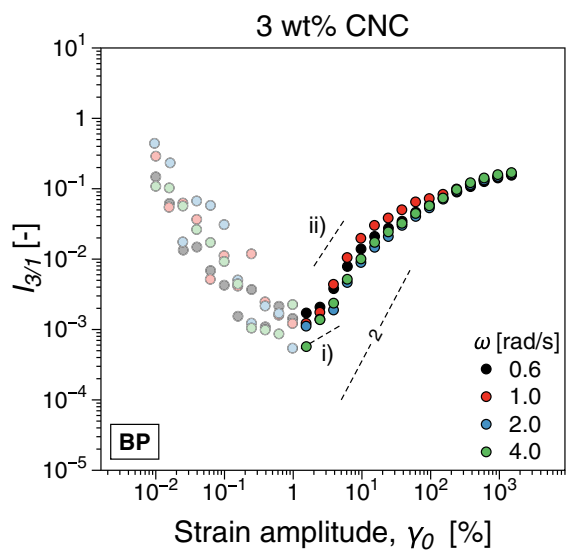

(b)

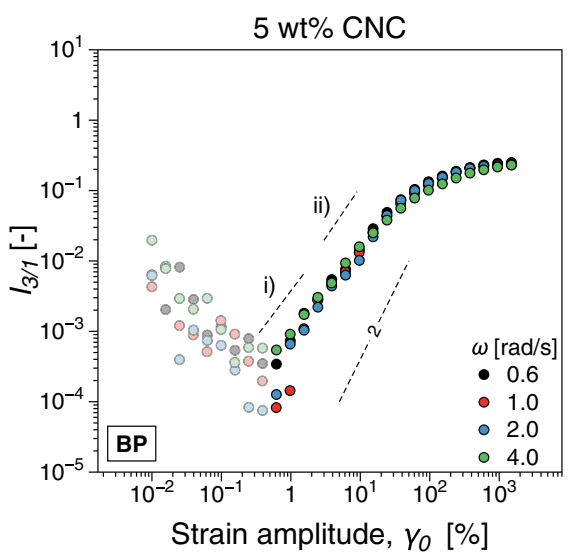

(d)

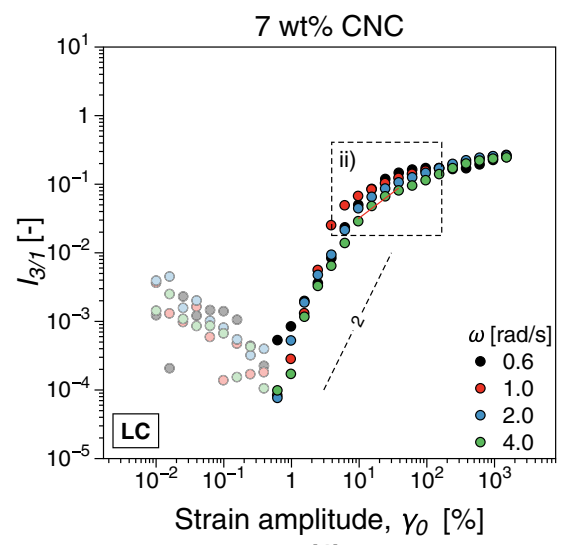

(f)
This behavior has been attributed to a weakly percolated network being disrupted by shear and confirmed through electrical conductivity measurements (Kádár et al. 2020b). For the CNC samples investigated, the existence of a weak gel network is confirmed by the linear viscoelastic dynamic moduli. Significantly low $\mathrm{CNC}$ concentrations lead to lower interaction between ions in aqueous suspension, which can be destroyed 
Fig. 6 (continued)

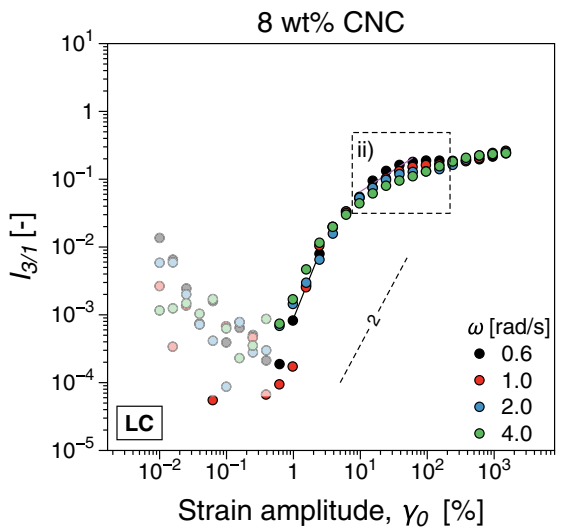

(g)

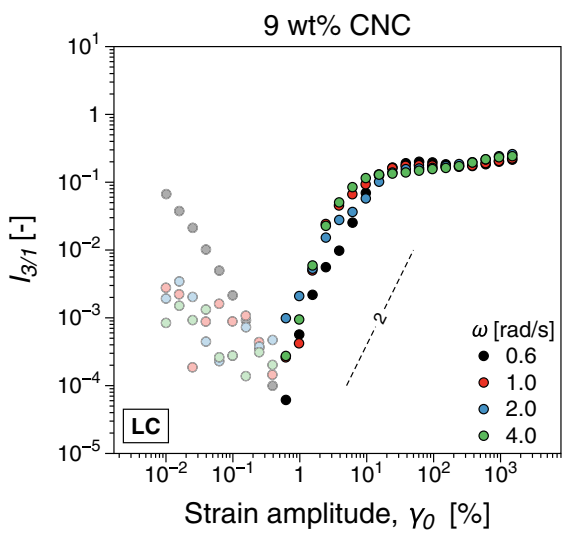

(h) while applying shear flow. We note here that such $\omega$ dependence seems to be inherent to the gel/percolation concentration, Kádár et al. (2020b), Meurer et al. (2021), which for other CNC systems may occur at higher concentrations than the isotropic-biphasic transition. In such a case, given its expected weakly elastic rheological behavior, a single quadratic scaling in the MAOS region would be expected for concentrations in the isotropic case, thus still being distinguishable from the other phases. For the biphasic 3-4 wt $\%$ CNC suspension, Fig. $6 \mathrm{~b}-\mathrm{c}$, a very weak angular frequency dependence was observed and was practically absent at $5 \mathrm{wt} \% \mathrm{CNC}$, Fig. 6d. Two scaling regions could be inferred for the biphasic 3-5 wt $\%$ : for $3 \mathrm{wt} \% \mathrm{CNC}$ (i) $n \approx 0.6$ for $\gamma_{0} \in[1,5] \%$ and (ii) $n \approx 1.5$ for $\gamma_{0} \in[5,10] \%$; for $4 \mathrm{wt} \%$ CNC: (i) $n \approx 1$ for $\gamma_{0} \in[0.7,3.8] \%$ and (ii) $n \approx 1.6$ for $\gamma_{0} \in[3.8,10]$ $\%$. The biphasic 5 wt $\%$ CNC suspension did not exhibit angular frequency dependency, but two distinct regions are observable at $\omega=0.6 \mathrm{rad} / \mathrm{s}$, however their characteristic slopes are nearly identical. This could be interpreted as a result of approaching the transition to the LC phase. For the liquid crystalline suspensions, 6-8 wt\% CNC, Fig. 6e-g in the range of strain amplitudes, where multiple scaling regions are generally identifiable, there is a clear quadratic scaling MAOS region, $n \approx 2$. Interestingly, a distinct $\omega-$ dependent region at the transition to LAOS could be inferred for 6-8 wt\% CNC.

Based on analysis above, we hypothesize that, two scaling regions (regions with different $n$ exponents, $I_{3 / 1} \propto \gamma_{0}^{n}$ ) with a significant angular frequency dependence is related to isotropic phase (2 wt\%) and originates from a weak gel-like behavior. In contrast, the biphasic 3-5 wt\% CNC, exhibited lower $\omega$ dependency, with two identifiable scaling regions. We note that the onset of the gel behavior does not necessarily correspond to the isotropic-biphasic transition, as the formation of tactoids can occur at lower or higher concentrations depending on the aspect ratio (Honorato-Rios et al. 2018). In addition, while the two scaling regions remained distinct for $\omega=0.6$ $\mathrm{rad} / \mathrm{s}$, their scaling was increasingly similar, signaling the transition to LC. Furthermore, a mostly quadratic MAOS region characterized the LC suspensions, 6-9 $\mathrm{wt} \% \mathrm{CNC}$, followed by a weak $\omega$-dependent region at the MAOS-LAOS transition. In turn, $\omega$ - dependency in the MAOS region could indicate, that LC 9 $\mathrm{wt} \%$ approaches a glassy state.

The elastic and viscous Lissajous-Bowditch (LB) diagrams for selected concentrations representative of the different CNC phases are given in Fig. 7. The LB diagrams are summarized in a Pipkin space which provides a "rheological fingerprint" that is the intra-cycle response as a function of the applied $\omega$ and $\gamma_{0}$. At low strain amplitudes, the stress response is affected by instrumentation noise, which is most observable for the isotropic phase due to the lower torque values measured, Fig. 7a. The elliptic curves, representing the linear viscoelastic response of the material, are observed at small strain amplitudes, see Fig. $7 \mathrm{a}$ for $\omega \leq 1 \mathrm{rad} / \mathrm{s}$ and $\gamma_{0}=1 \%$. As the strain amplitude increases, the LB loops appear to be increasingly distorted, which indicates the occurrence of intra-cycle nonlinearities. From qualitative point of view, the isotropic LB diagrams, 7b, stand out compared to the biphasic and the liquid crystalline phase LB diagrams, $7 \mathrm{c}$, that are comparatively 

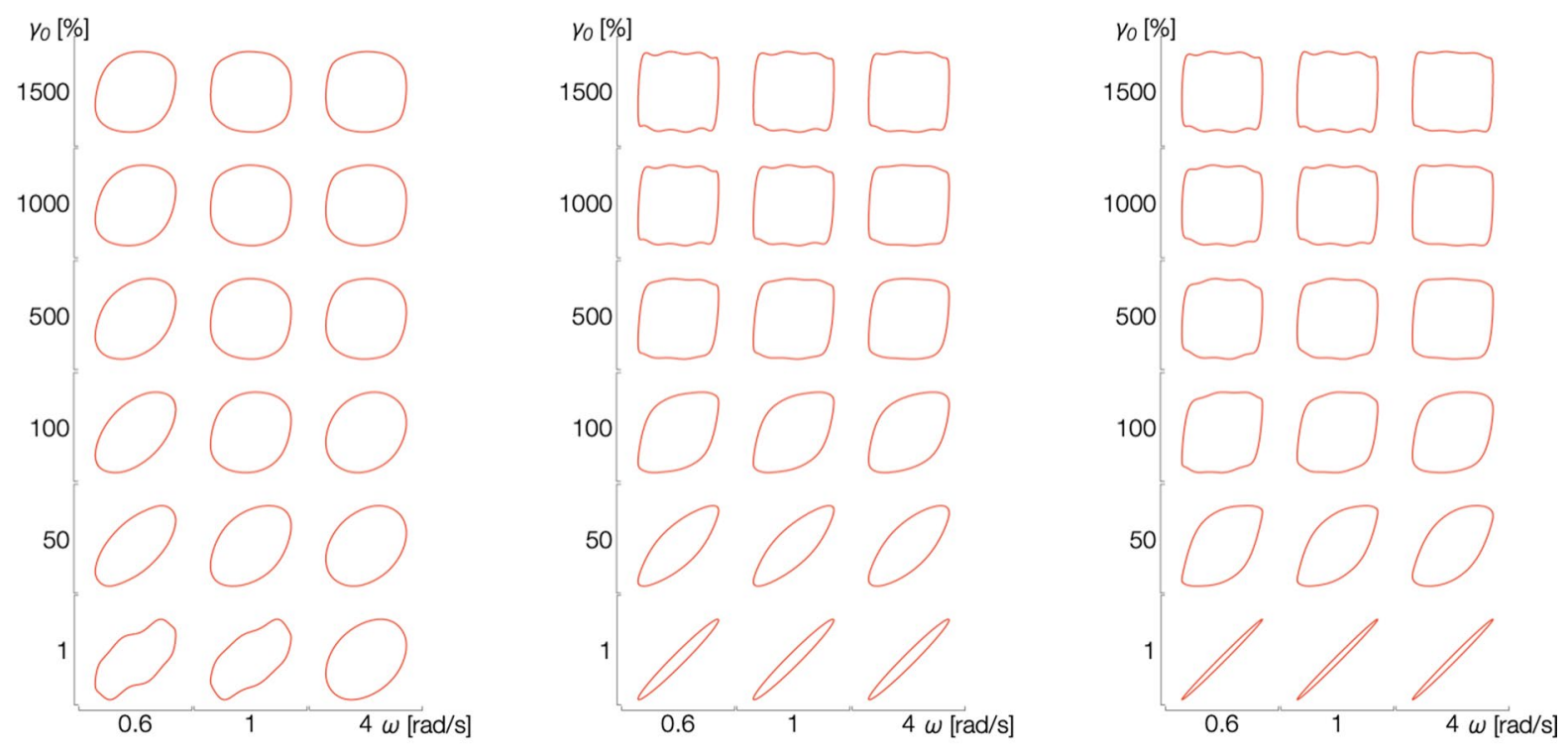

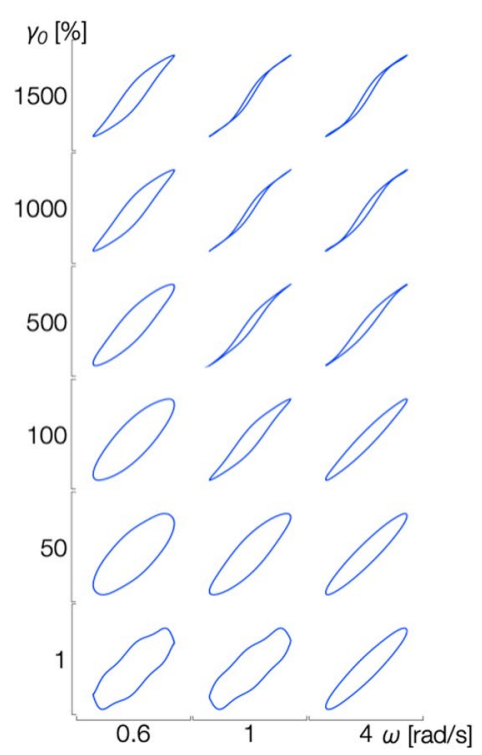

(a)

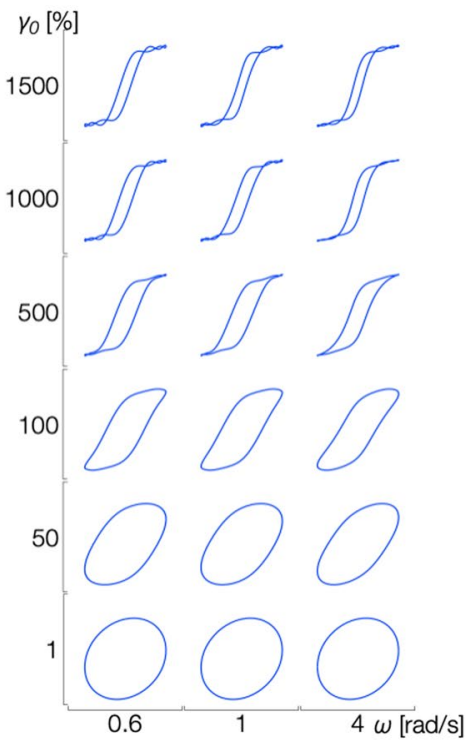

(b)

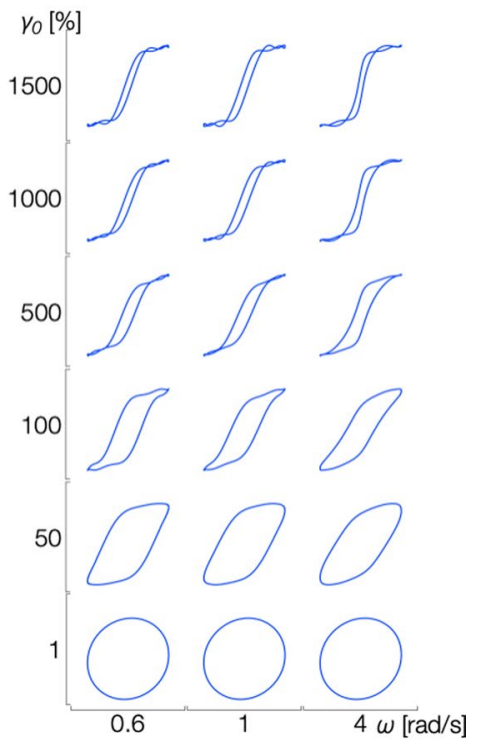

(c)
Fig. 7 Lissajous-Bowditch (LB) diagrams at selected $\omega$ and $\gamma_{0}$ for CNC concentrations representative of different phases studied: a isotropic ( $2 \mathrm{wt} \%$ ), b biphasic (5 wt\%), c liquid crys-

more similar to each other. The shape of the loop also depends on the imposed $\omega$, most readily observable for $2 \mathrm{wt} \% \mathrm{CNC}$, Fig. 7a, as expected. This is in agreement with the LVE dynamic moduli and $I_{3 / 1}$ results, pointing towards the existence of a weakly gelled structure. We note that the viscous LB diagrams talline (7 wt \%). The top row contains the elastic LB diagrams (red) and bottom row contains the viscous LB diagrams (blue)

show self-intersecting loops for the biphasic and liquid crystalline phases. Self-intersecting loops have also been observed by Moud et al. (2021) for highly concentrated hydrogels with cellulose nanocrystals and was attributed to an overshoot in shear stress response. 
Fig. 8 Strain-stiffening, $S$, and shear thickening, $T$, nonlinear material parameters: a $2 \mathrm{wt} \%$, b $3 \mathrm{wt} \%$, c $4 \mathrm{wt} \%$, d $5 \mathrm{wt} \%$, e $6 \mathrm{wt} \%$, f $7 \mathrm{wt} \%, \mathbf{g} 8 \mathrm{wt} \%, \mathbf{h} 9 \mathrm{wt} \%$ $\mathrm{CNC}$

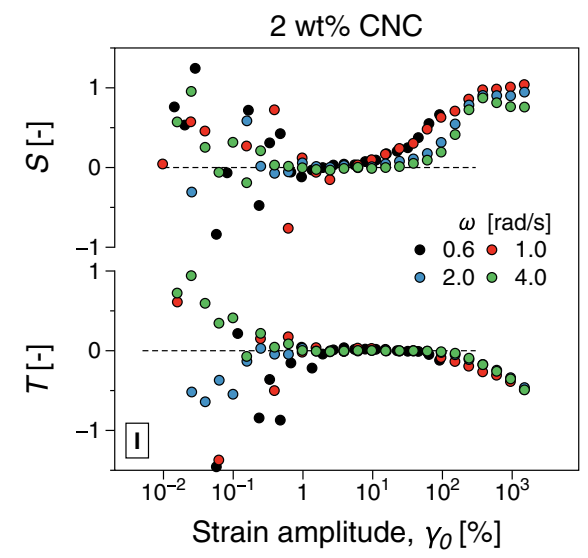

(a)

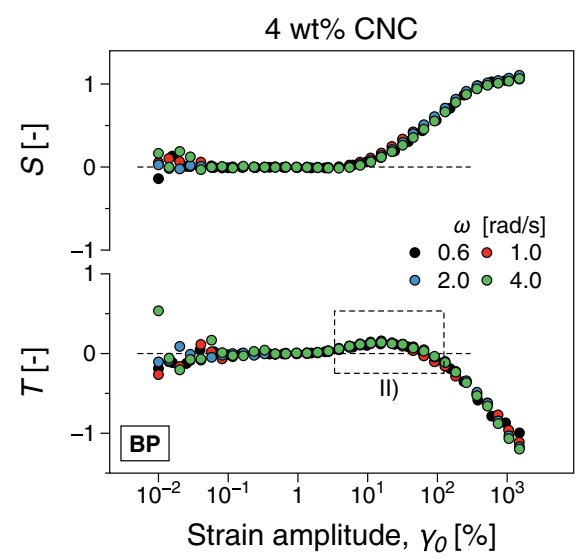

(c)

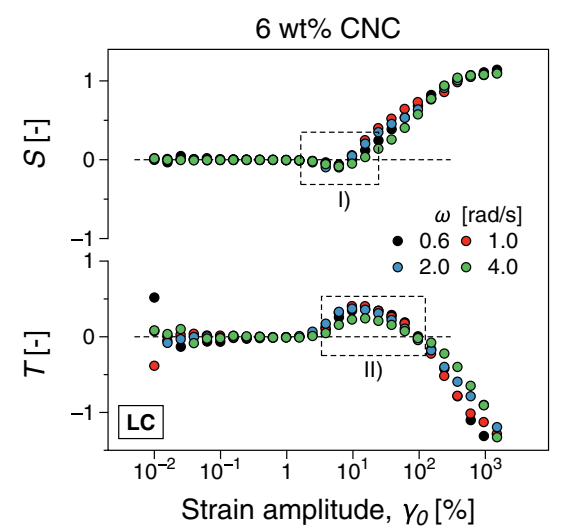

(e)

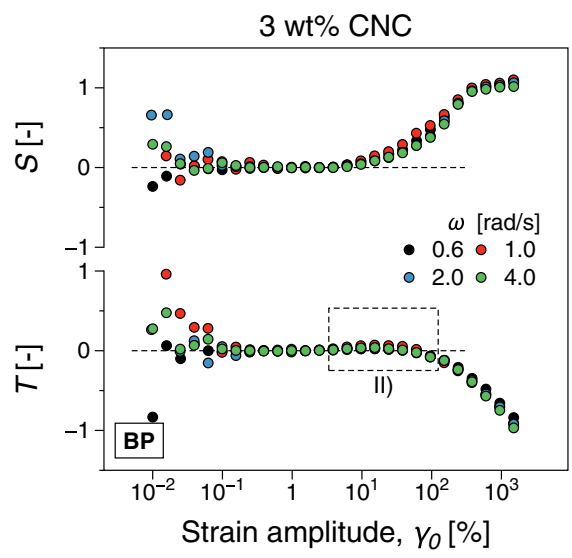

(b)

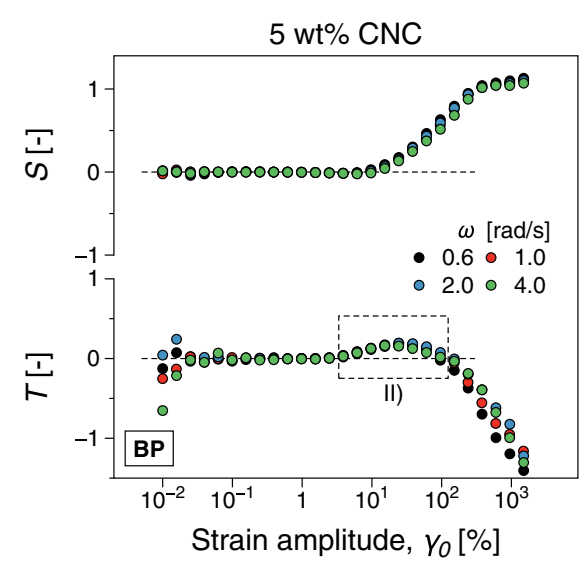

(d)

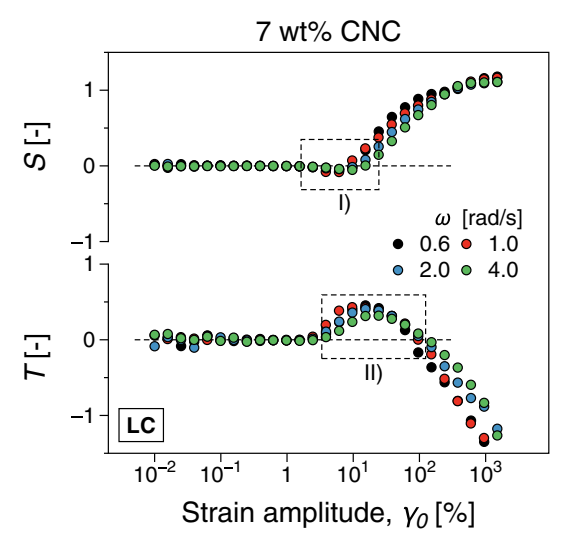

(f)
The strain-stiffening $(S)$ and shear-thickening $(T)$ dimensionless ratios are compiled in Fig. 8 for all investigated concentrations. As expected, at low strain amplitudes, within the linear viscoelastic region, $S, T \cong 0$. The lowest CNC concentration, $2 \mathrm{wt} \%$, due to its low viscosity, was affected by a low torque, resulting in scattered datapoints at low $\gamma_{0}$, Fig. 8a.

All suspensions exhibit a dominant intra-cycle strain-stiffening nonlinear behavior, where $S>0$, independently on applied $\omega$. There is also a notable 
Fig. 8 (continued)

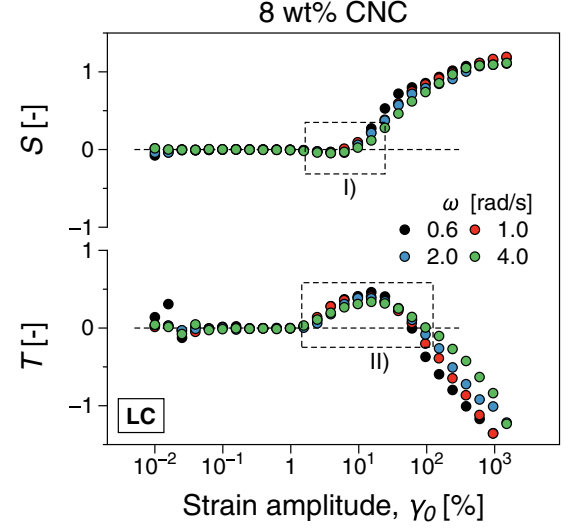

(g)

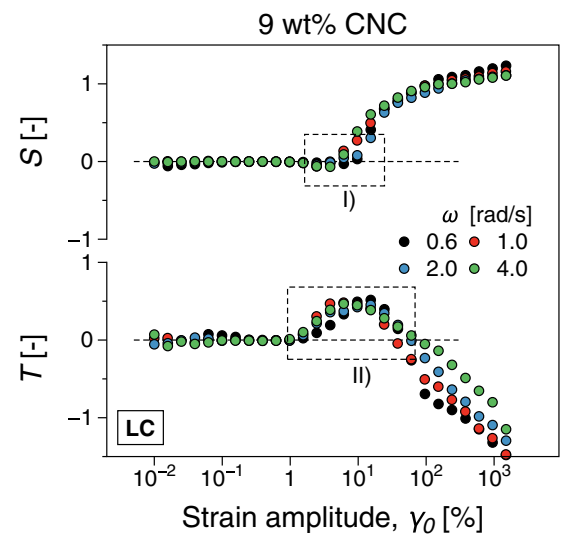

(h) increase in strain-stiffening between the lowest and the highest $\mathrm{CNC}$ concentrations, i.e. compare Fig. 8a with $8 \mathrm{~h}$. We note that the positive sign in $S$ is an apparent stiffening because at large strain amplitudes both $G_{L}^{\prime}, G_{M}^{\prime}$ soften, see Eq. 3 and Fig. S3, however the nonlinear response is dominated by $G_{M}^{\prime}$ softening more quickly than $G_{L}^{\prime}$ (Khandavalli and Rothstein 2015). For the isotropic $2 \mathrm{wt} \% \mathrm{CNC} S$ is a function of the applied $\omega$ in the nonlinear region, mirroring the $G^{\prime}, G^{\prime \prime}$ and $I_{3 / 1}$ behavior. Interestingly, for the 6-9 wt\% CNC suspensions there is a slight but persistent $S<0$ region, see I) in Fig. 8e-h, i.e. a local intra-cycle strain softening behavior, around $\gamma_{0}=2 \%$ before transitioning to $S>0$ with increasing $\gamma_{0}$.

For all investigated concentrations, a dominant nonlinear viscous intra-cycle shear-thinning behavior is observed, where $T<0$. Interestingly, the $\omega$ -dependence, that has been observed in $G^{\prime}, G^{\prime \prime}$ and $I_{3 / 1}$, is mostly limited to the strain stiffening ratio $S$ in the stress decomposition analysis. At $3 \mathrm{wt} \% \mathrm{CNC}$, we noted a slight $T>0$ region, see II) in Fig. 8b, i.e. a local intra-cycle shear-thickening behavior region around $10^{1} \%$, whereafter $T$ drops, resulting in intracycle shear-thinning, $T<0$. By comparing the relative magnitude of $\eta_{L}^{\prime}$ and $\eta_{M}^{\prime}$, see Fig. S4 in the Supplementary Information, it can be concluded that they both experience a local thickening before thinning at large strain amplitudes. The strain-rate thickening behavior, where $T>0$, at medium strain amplitudes was caused by stronger nonlinearity of $\eta_{L}^{\prime}$ than $\eta_{M}^{\prime}$. In turn, a negative sign in $T$ at increased strain amplitude was dominated by $\eta_{M}^{\prime}$ thinning more quickly than $\eta_{L}^{\prime}$ (Khandavalli and Rothstein 2015). This behavior is more readily observable for concentrations $>3$ wt $\%$ CNC, Fig. $8 \mathrm{c}-\mathrm{h}$, with the $T>0$ region covering approximately 1 decade of strain amplitude for the highest investigated concentration, $9 \% \mathrm{CNC}$, Fig. $8 \mathrm{~h}$. Local intra-cycle shear-thickening behavior has also been observed in other complex fluid systems (Khandavalli and Rothstein 2015; Sun et al. 2011). A dual behavior, where at lower strain amplitude intra-cycle shear-thickening region is observed, could indicate jamming of the gel-like microstructure, while intracycle shear-thinning at higher strain amplitude could be associated with the orientation or yielding of CNC particles in the flow direction (Wagner and Brady 2009; Kamibayashi et al. 2008). Since the behavior is more prominent with increasing CNC concentration from the biphasic region onward, it is apparent that the liquid crystalline domains play a major role in the microstructural jamming. The 6-9 wt\% CNC suspensions exhibited an apparent dependence on the imposed angular frequency at very high strain amplitudes $\left(\gamma_{0}>100 \%\right)$. We assume that this could be an artifact affected by the self-intersecting visible in LB diagrams at the same $\omega$.

Based on stress decomposition analysis, we hypothesize that, similarly to $I_{3 / 1}$, a significant angular frequency dependence is related to the isotropic phase ( $2 \mathrm{wt} \% \mathrm{CNC}$ ) and relates to the weak gel-like network present at that concentration. In turn, a dual behavior exhibited by the $T$ parameter, where at medium strain amplitudes $T>0$ and at large strain amplitude $T<0$, can indicate the presence of a biphasic phase for 3-5 wt $\%$ aqueous CNC suspensions. Furthermore, the intra-cycle strain softening behavior 
at medium strain amplitude, where $S<0$, effectively marks the transition from the biphasic to the liquid crystalline phase for 6-9 wt\% CNC. Finally, we must also note that the present analysis cannot exclude the existence of slip artefacts (Hubbe et al. 2017). Wall slip has been evidenced in nonlinear oscillatory shear tests for crosslinked hydrogels (Kamkar et al. 2021). Interestingly, in the scientific literature concentrations higher than investigated in this study, e.g. 10 wt\% CNC, were found not to exhibit wall slip (Derakhshandeh et al. 2013). Danesh et al. (2021) showed that concentrations $1-5 \mathrm{wt} \% \mathrm{CNC}$ obeyed the noslip boundary condition, while the addition of $\mathrm{NaCl}$ caused wall slip.
Rheo-PLI in oscillatory shear

The PLI space time-diagrams corresponding to strain sweep tests at $\omega=0.6 \mathrm{rad} / \mathrm{s}$ are shown in Fig. 9, with individual cycles from selected strain amplitudes shown in Fig. 10. The same dataset for $\omega=4 \mathrm{rad} / \mathrm{s}$ can be found in Figs. S5 and S6 in the Supplementary information. The onset of nonlinear viscoelastic behavior is overlaid on the images by marking the strain amplitudes for which $S, T$ are non-zero.

We briefly note that a quantitative image analysis would not be reliable due to variable illumination conditions during and between the tests and that the onset of nonlinear behavior is a strong function of the data analysis method, with the onset differing between the linear viscoelastic dynamic moduli, $I_{3 / 1}$ and $S, T$ (Meurer et al. 2021). Overall, however, linear viscoelastic behavior generally corresponds to
Fig. 9 Space-time optical visualizations of the birefringence pattern development and phase transitions in dynamic strain sweep measurements at $\omega=0.6$ $\mathrm{rad} / \mathrm{s}$

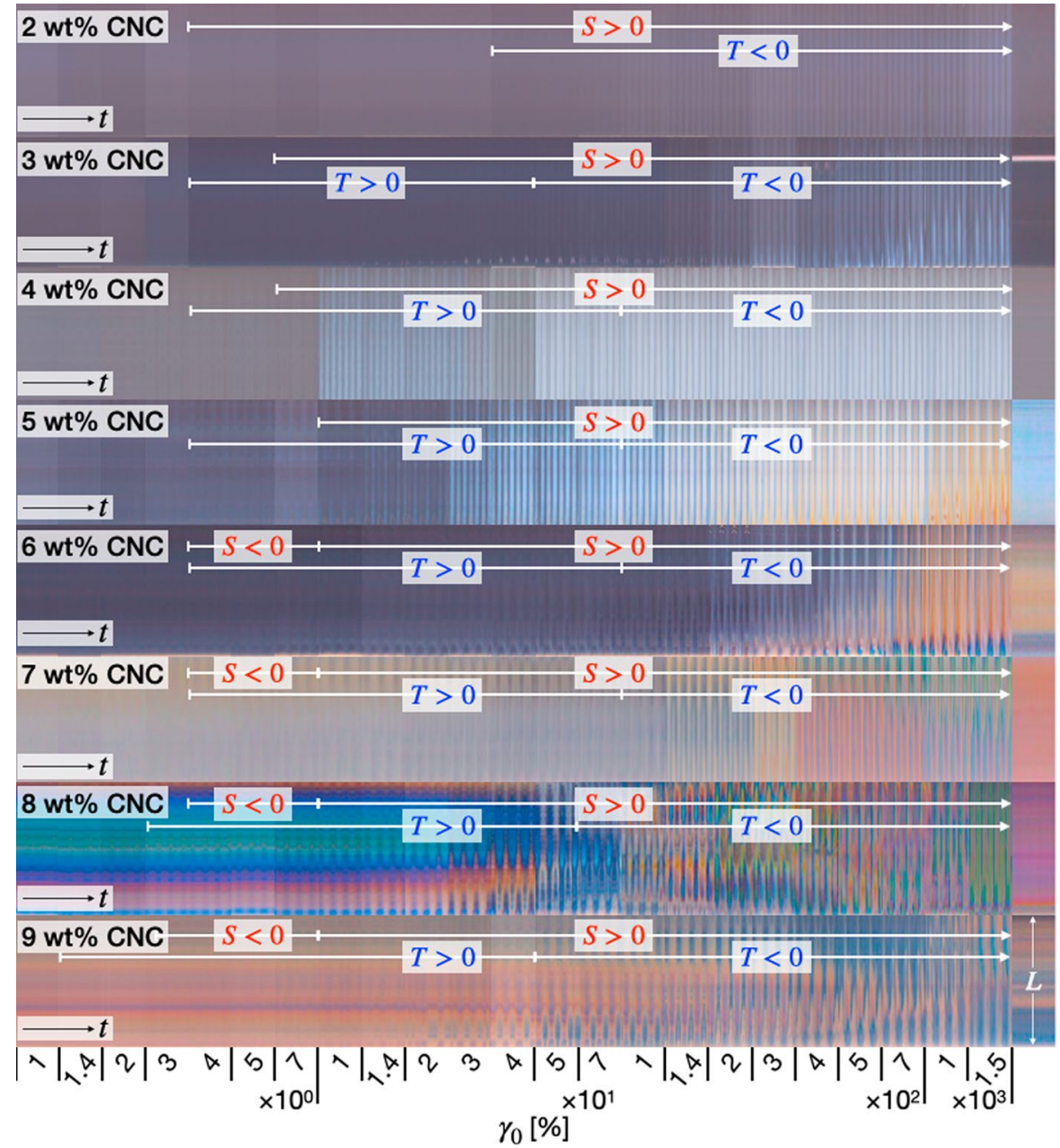




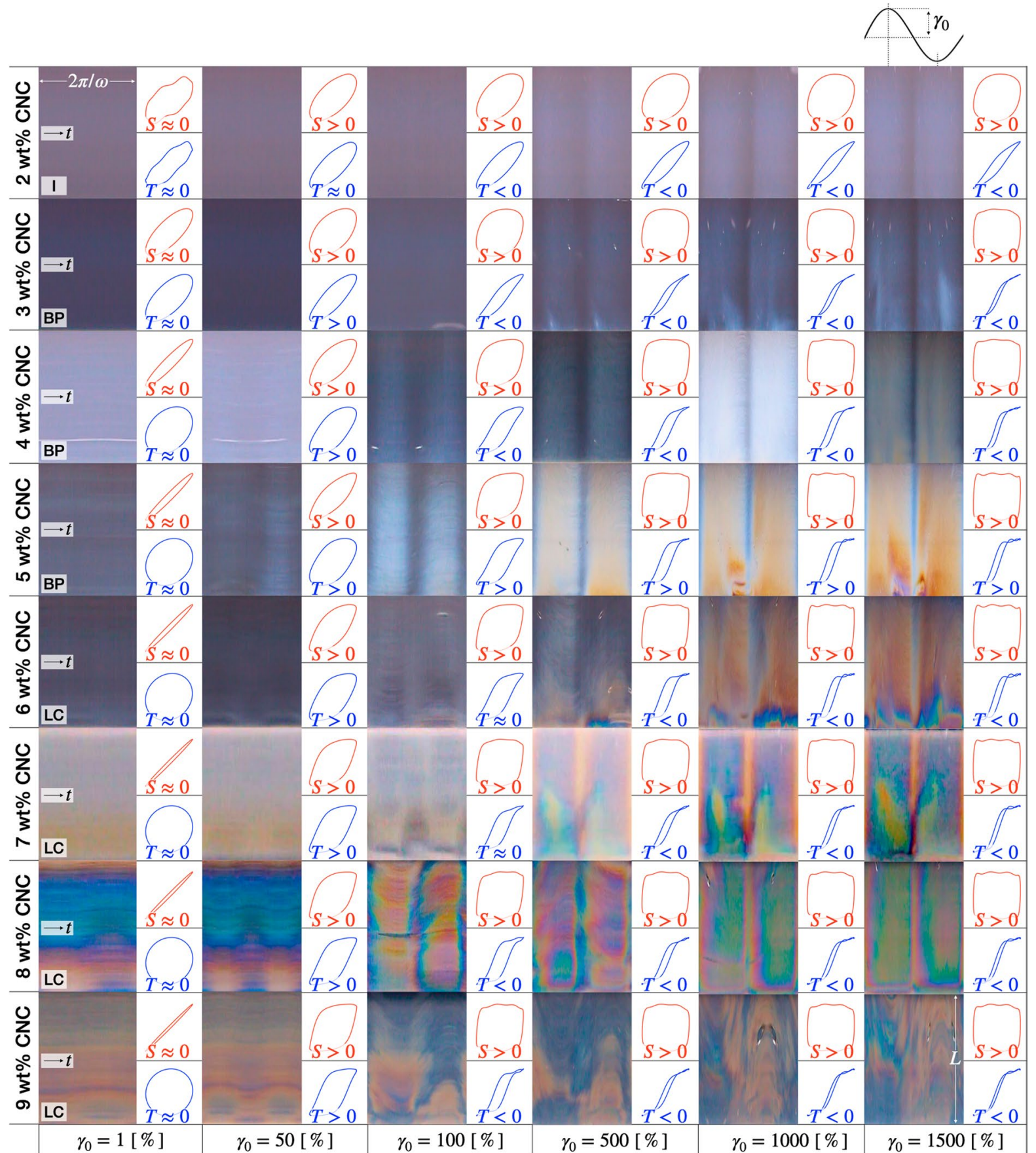

Fig. 10 Intra-cycle birefringence patterns at selected strain amplitudes for $\omega=0.6 \mathrm{rad} / \mathrm{s}$, corresponding to the space-time diagrams in Fig. 9. The corresponding elastic and viscous LB diagrams, see also Fig. 7, are also included

no observable birefringence effects, e.g. see $\gamma_{0}=1 \%$ in Fig. 10, except at the higher end of the concentration range, 8-9 wt \%, where there is a significantly higher density of the mesophase while approaching the glassy state. Interestingly, within the $T$ overshoot region, see especially $\gamma_{0}=50 \%$ in Fig. 10, although intra-cycle birefringence variations are observable there are no significant color distortions. This 
supports the premise that the local intra-cycle shearthickening behavior is determined by microstructural jamming with no major structural re-arrangements. For increasing strain amplitudes and concentrations, increasingly significant intra-cycle color distortions are observed, corresponding to $S>0$ and $T<0$, Fig. 10. The distortions are induced from the free surface of the flow domain (lower end of the images) and propagate inwards, as the local shear rates are proportional to the radial position in plate-plate flow (Kádár et al. 2020a). It is apparent that the color progression within a cycle could mimic the elastic LB diagrams, e.g. compare $5 \mathrm{wt} \% \mathrm{CNC} \gamma_{0}=100 \%$ and $\gamma_{0}=500 \%$ in Fig. 10: a more gradual color progression appears to corresponds to smoother more elliptic shaped elastic LB, whereas a more abrupt progression corresponds to more square-like elastic LBs. Likewise, for high strain amplitudes and CNC concentrations, intra-cycle color asymmetries are recorded that could be contributing factors to the oscillations recorded around the minimum strain in elastic LB diagrams and the self-intersecting loops in the viscous LB diagrams, see also Fig. 7. Interestingly, at the onset of the MAOS region in $I_{3 / 1}$ no significant intra-cycle color variations were recorded, suggesting that the nonlinear behavior originate from both the isotropic parts of biphasic suspensions and interfaces between mesogens in the LC phase, as well as, with increasing strain amplitudes, changes in the orientation of the liquid crystalline domains (Fazilati et al. 2021).

\section{Conclusions}

The linear and nonlinear viscoelasticity of isotropic (2 wt\% CNC), biphasic (3-5 wt\% $\mathrm{CNC}$ ) and liquid crystalline $(\geq 6 \mathrm{wt} \%)$ phases in $\mathrm{CNC}$ water suspensions were investigated in this study. The phases were determined based on a combined analysis of polarized optical microscopy, linear viscoelastic oscillatory shear, steady shear viscosity functions and combined rheo-PLI experiments. The results were then compared with Fourier-transform Rheology and stress decomposition analysis to determine the influence of the particular CNC phase on the nonlinear material parameters. The relationship between the $\mathrm{CNC}$ phase and the rheological parameters investigated is summarized in Table S3 and Fig. S7 in the Supplementary information. The intra-cycle and inter-cycle nonlinear material parameters investigated in this study were capable of identifying all CNC phases, with changes in $I_{3 / 1}$ scaling behavior with $\gamma_{0}$ distinguishing all investigated phases, the shear-thickening overshoot in $T$ identifying biphasic suspensions, and a local slight strain-stiffening in $S$ appeared to be associated only with the liquid crystalline phase. Therefore, based on the present results it can be concluded that nonlinear materials parameters from FT rheology and stress decomposition could be a more sensitive framework to identify $\mathrm{CNC}$ suspensions phases from rheological tests.

Acknowledgments The authors are grateful for the financial support of the Wallenberg Wood Science Centre (SW, TN, GW, RK) and of the Chalmers Area of Advance Materials Science (MF, TN, RK). The authors also thank Jinhua Sun for the helpful instructions on using the Zetasizer Nano ZS.

Funding Open access funding provided by Chalmers University of Technology. The authors have not disclosed any funding.

\section{Declarations}

Conflict of interest The authors declare that they have no conflict of interest.

Open Access This article is licensed under a Creative Commons Attribution 4.0 International License, which permits use, sharing, adaptation, distribution and reproduction in any medium or format, as long as you give appropriate credit to the original author(s) and the source, provide a link to the Creative Commons licence, and indicate if changes were made. The images or other third party material in this article are included in the article's Creative Commons licence, unless indicated otherwise in a credit line to the material. If material is not included in the article's Creative Commons licence and your intended use is not permitted by statutory regulation or exceeds the permitted use, you will need to obtain permission directly from the copyright holder. To view a copy of this licence, visit http://creativecommons.org/licenses/by/4.0/.

\section{References}

Abbasi Moud A, Kamkar M, Sanati-Nezhad A, Hejazi SH, Sundararaj U (2020) Nonlinear viscoelastic characterization of charged cellulose nanocrystal network structure in the presence of salt in aqueous media. Cellulose 27(10):5729-5743. https://doi.org/10.1007/ s10570-020-03166-x

Abitbol T, Cranston ED (2014) Chiral nematic self-assembly of cellulose nanocrystals in suspensions and solid films. Handbook of green materials, vol 37. World Scientific, 
Singapore, pp 37-56. https://doi.org/10.1142/9789814566 469_0035

Abitbol T, Kam D, Levi-Kalisman Y, Gray DG, Shoseyov O (2018) Surface charge influence on the phase separation and viscosity of cellulose nanocrystals. Langmuir 34(13):3925-3933. https://doi.org/10.1021/acs.langmuir. $7 \mathrm{~b} 04127$

Chen Y, Xu C, Huang J, Wu D, Lv Q (2017) Rheological properties of nanocrystalline cellulose suspensions. Carbohydr Polym 157:303-310. https://doi.org/10.1016/j.carbpol. 2016.10.002

Conley K, Whitehead MA, van de Ven TGM (2017) Probing the structural chirality of crystalline cellulose with induced circular dichroism. Cellulose 24(2):479-486. https://doi.org/10.1007/s10570-016-1130-8

Danesh M, Moud AA, Mauran D, Hojabr S, Berry R, Pawlik M, Hatzikiriakos SG (2021) The yielding of attractive gels of nanocrystal cellulose (CNC). J Rheol 65(5):855-869. https://doi.org/10.1122/8.0000247

Derakhshandeh B, Petekidis G, Shafiei Sabet S, Hamad WY, Hatzikiriakos SG (2013) Ageing, yielding, and rheology of nanocrystalline cellulose suspensions. J Rheol 57(1):131-148. https://doi.org/10.1122/1.4764080

Dong XM, Kimura T, Revol JF, Gray DG (1996) Effects of ionic strength on the isotropic-chiral nematic phase transition of suspensions of cellulose crystallites. Langmuir 12(8):2076-2082. https://doi.org/10.1021/la950133b

Dong XM, Revol JF, Gray DG (1998) Effect of microcrystallite preparation conditions on the formation of colloid crystals of cellulose. Cellulose 5(1):19-32. https://doi.org/10. 1023/A:1009260511939

Dufresne A (2012) Nanocellulose: from nature to high performance tailored materials. De Gruyter, Berlin. https://doi. org/10.1515/9783110254600

Dumanli AG, van der Kooij HM, Kamita G, Reisner E, Baumberg JJ, Steiner U, Vignolini S (2014) Digital color in cellulose nanocrystal films. ACS Appl Mater Interfaces 6(15):12302-12306. https://doi.org/10.1021/am501995e

Ewoldt RA, Hosoi A, McKinley G (2008) New measures for characterizing nonlinear viscoelasticity in large amplitude oscillatory shear. J Rheol 52(1427):1427-1458. https:// doi.org/10.1122/1.2970095

Fazilati M, Ingelsten S, Wojno S, Nypelö T, Kádár R (2021) Thixotropy of cellulose nanocrystal suspensions. J Rheol. https://doi.org/10.1122/8.0000281

Fernandes SN, Almeida PL, Monge N, Aguirre LE, Reis D, de Oliveira CLP, Neto AMF, Pieranski P, Godinho MH (2017) Mind the microgap in iridescent cellulose nanocrystal films. Adv Mater 29(2):1603560. https://doi. org/10.1002/adma.201770008

Gaska K, Kádár R (2019) Evidence of percolated network at the linear-nonlinear transition in oscillatory shear. AIP Conf Proc 2107(1):050003. https://doi.org/10.1063/1. 5109509

Habibi Y, Lucia LA, Rojas OJ (2010) Cellulose nanocrystals: chemistry, self-assembly, and applications. Chem Rev 110(6):3479-3500. https://doi.org/10.1021/cr900339w

Haniffa MACM, Ching YC, Abdullah LC, Poh SC, Chuah CH (2016) Review of bionanocomposite coating films and their applications. Polymers 8(7):246. https://doi.org/10. 3390/polym8070246
Hausmann MK, Rühs PA, Siqueira G, Läuger J, Libanori R, Zimmermann T, Studart AR (2018) Dynamics of cellulose nanocrystal alignment during $3 \mathrm{~d}$ printing. ACS Nano 12(7):6926-6937. https://doi.org/10.1021/acsnano.8b023 66

Haywood AD, Weigandt KM, Saha P, Noor M, Green MJ, Davis VA (2017) New insights into the flow and microstructural relaxation behavior of biphasic cellulose nanocrystal dispersions from rheosans. Soft Matter 13:8451-8462. https://doi.org/10.1039/C7SM00685C

Honorato-Rios C, Lehr C, Schütz C, Sanctuary R, Osipov MA, Baller J, Lagerwall JPF (2018) Fractionation of cellulose nanocrystals: enhancing liquid crystal ordering without promoting gelation. NPG Asia Mater 10(5):455-465. https://doi.org/10.1038/s41427-018-0046-1

Hubbe MA, Pegah T, Joyce M, Tyagi P, Kehoe M, DimicMisic K, Pal L (2017) Rheology of nanocelluloserich aqueous suspensions: a review. BioResources 12(4):9556-9661. https://doi.org/10.15376/biores.12.4. Hubbe

Hyun K, Kim W (2011) A new non-linear parameter q from ft-rheology under nonlinear dynamic oscillatory shear for polymer melts system. Korea-Aust Rheol J 23(4):227235. https://doi.org/10.1007/s13367-011-0028-0

Hyun K, Baik ES, Ahn KH, Lee SJ, Sugimoto M, Koyama K (2007) Fourier-transform rheology under medium amplitude oscillatory shear for linear and branched polymer melts. J Rheol 51(6):1319-1342. https://doi.org/10. $1122 / 1.2790072$

Hyun K, Wilhelm M, Klein CO, Cho KS, Nam JG, Ahn KH, Lee SJ, Ewoldt RH, McKinley GH (2011) A review of nonlinear oscillatory shear tests: analysis and application of large amplitude oscillatory shear (LAOS). Prog Polym Sci 36(12):1697-1753. https://doi.org/10.1016/j.progp olymsci.2011.02.002

Kádár R, Abbasi M, Figuli R, Rigdahl M, Wilhelm M (2017) Linear and nonlinear rheology combined with dielectric spectroscopy of hybrid polymer nanocomposites for semiconductive applications. Nanomaterials 7(2):23. https:// doi.org/10.3390/nano7020023

Kádár R, Fazilati M, Nypelö T (2020) Unexpected microphase transitions in flow towards nematic order of cellulose nanocrystals. Cellulose 27(4):2003-2014. https://doi.org/ 10.1007/s10570-019-02888-x

Kádár R, Gaska K, Gkourmpis T (2020) Nonlinear oddities at the percolation of $3 \mathrm{~d}$ hierarchical graphene polymer nanocomposites. Rheol Acta 59(5):333-347. https://doi.org/10. 1007/s00397-020-01204-w

Kádár R, Spirk S, Nypelö T (2021) Cellulose nanocrystal liquid crystal phases: Progress and challenges in characterization using rheology coupled to optics, scattering, and spectroscopy. ACS Nano 15(5):7931-7945. https://doi. org/10.1021/acsnano.0c09829

Kamibayashi M, Ogura H, Otsubo Y (2008) Shear-thickening flow of nanoparticle suspensions flocculated by polymer bridging. J Colloid Interface Sci 321(2):294-301. https:// doi.org/10.1016/j.jcis.2008.02.022

Kamkar M, Janmaleki M, Erfanian E, Sanati-Nezhad A, Sundararaj U (2021) Viscoelastic behavior of covalently 
crosslinked hydrogels under large shear deformations: an approach to eliminate wall slip. Phys Fluids 33(4):041702. https://doi.org/10.1063/5.0046801

Khandavalli S, Rothstein JP (2015) Large amplitude oscillatory shear rheology of three different shear-thickening particle dispersions. Rheol Acta 54:601-618. https://doi.org/10. 1007/s00397-015-0855-x

Klemm D, Kramer F, Moritz S, Lindström T, Ankerfors M, Gray D, Dorris A (2011) Nanocelluloses: a new family of nature-based materials. Angew Chem Int Ed 50(24):54385466. https://doi.org/10.1002/anie.201001273

Marchessault RH, Morehead FF, Walter NM (1959) Liquid crystal systems from fibrillar polysaccharides. Nature 184(4686):632-633. https://doi.org/10.1038/184632a0

Meurer M, Kádár R, Ramakers-van Dorp E, Möginger B, Hausnerova B (2021) Nonlinear oscillatory shear tests of pressure sensitive adhesives (psa) designed for transdermal therapeutic systems (tts). Rheol Acta 60:553-570. https://doi.org/10.1007/s00397-021-01280-6

Moud AA, Kamkar M, Sanati-Nezhad A, Hejazi SH, Sundararaj U (2021) Viscoelastic properties of poly (vinyl alcohol) hydrogels with cellulose nanocrystals fabricated through sodium chloride addition: Rheological evidence of double network formation. Colloids Surf A 609:125577. https:// doi.org/10.1016/j.colsurfa.2020.125577

Natalia I, Ewoldt RH, Koos E (2020) Questioning a fundamental assumption of rheology: Observation of noninteger power expansions. J Rheol 64(3):625-635. https://doi.org/ 10.1122/1.5130707

Navarro SL, Nakayama K, Idström A, Evenäs L, Ström A, Nypelö T (2021) The effect of sulfate half-ester groups on cellulose nanocrystal periodate oxidation. Cellulose 28(15):9633-9644. https://doi.org/10.1007/ s10570-021-04115-y

Onogi S, Asada T (1980) Principles, chapter rheology and rheo-optics of polymer liquid crystals. In: Rheology, vol. 1, Springer, Boston

Onsager L (1949) The effects of shape on the interaction of colloidal particles. Ann N Y Acad Sci 51(4):627-659. https://doi.org/10.1111/j.1749-6632.1949.tb27296.x

Orts WJ, Godbout L, Marchessault RH, Revol JF (1998) Enhanced ordering of liquid crystalline suspensions of cellulose microfibrils: A small angle neutron scattering study. Macromolecules 31(17):5117-5725. https://doi.org/ 10.1021/ma9711452

Pignon F, Challamel M, De Geyer A, Elchamaa M, Semeraro EF, Hengl N, Jean B, Putaux JL, Gicquel E, Bras J, Prevost S, Sztucki M, Narayanan T, Djeridi H (2021) Breakdown and buildup mechanisms of cellulose nanocrystal suspensions under shear and upon relaxation probed by saxs and sals. Carbohydr Polym 260:117751. https://doi. org/10.1016/j.carbpol.2021.117751

Qing Y, Sabo R, Zhu J, Agarwal U, Cai Z, Wu Y (2013) A comparative study of cellulose nanofibrils disintegrated via multiple processing approaches. Carbohydr Polym 97(1):226-234. https://doi.org/10.1016/j.carbpol.2013.04. 086

Rånby B, Banderet A, Sillén LG (1949) Aqueous colloidal solutions of cellulose micelles. Acta Chem Scand 3:649650. https://doi.org/10.3891/acta.chem.scand.03-0649
Revol JF, Bradford H, Giasson J, Marchessault R, Gray D (1992) Helicoidal self-ordering of cellulose microfibrils in aqueous suspension. Int J Biol Macromol 14(3):170-172. https://doi.org/10.1016/S0141-8130(05)80008-X

Saito T, Hirota M, Tamura N, Kimura S, Fukuzumi H, Heux L, Isogai A (2009) Individualization of nano-sized plant cellulose fibrils by direct surface carboxylation using tempo catalyst under neutral conditions. Biomacromolecules 10(7):1992-1996. https://doi.org/10.1021/bm900414t

Shafeiei-Sabet S, Hamad WY, Hatzikiriakos SG (2013) Influence of degree of sulfation on the rheology of cellulose nanocrystal suspensions. Rheol Acta 52(8):741-751. https://doi.org/10.1007/s00397-013-0722-6

Shafiei-Sabet S, Hamad WY, Hatzikiriakos SG (2012) Rheology of nanocrystalline cellulose aqueous suspensions. Langmuir 28(49):17124-17133. https://doi.org/10.1021/ la303380v

Sun W, Yang Y, Wang T, Liu X, Wang C, Tong Z (2011) Large amplitude oscillatory shear rheology for nonlinear viscoelasticity in hectorite suspensions containing poly(ethylene glycol). Polymer 52(6):1402-1409. https://doi.org/10. 1016/j.polymer.2011.01.048

Ureña-Benavides EE, Ao G, Davis VA, Kitchens CL (2011) Rheology and phase behavior of lyotropic cellulose nanocrystal suspensions. Macromolecules 44(22):89908998. https://doi.org/10.1021/ma201649f

Van den Berg MEH, Kuster S, Windhab EJ, Sagis LMC, Fischer P (2018) Nonlinear shear and dilatational rheology of viscoelastic interfacial layers of cellulose nanocrystals. Phys Fluids 30(7):072103. https://doi.org/10.1063/1. 5035334

Völker-Pop L (2014) Optical methods in rheology: polarized light imaging. Chem Listy 108:697-724

Wagner N, Brady J (2009) Shear thickening in colloidal dispersions. Phys Today 62:27-32. https://doi.org/10.1063/1. 3248476

Wilhelm M, van Dusschoten D (2001) Increased torque transducer sensitivity via oversampling. Rheol Acta 40:395399. https://doi.org/10.1007/s003970000158

Wojno S, Westman G, Kádár R (2019) Gel point in CNC dispersion from FT rheology. Annu Trans Nordic Rheol Soc 27:179-184

Xu Y, Atrens AD, Stokes JR (2018) Liquid, gel and soft glass phase transitions and rheology of nanocrystalline cellulose suspensions as a function of concentration and salinity. Soft Matter 14:1953-1963. https://doi.org/10.1039/ C7SM02470C

Zhang Y, Cheng Q, Chang C, Zhang L (2018) Phase transition identification of cellulose nanocrystal suspensions derived from various raw materials. J Appl Polym Sci 135(24):45702. https://doi.org/10.1002/app.45702

Publisher's Note Springer Nature remains neutral with regard to jurisdictional claims in published maps and institutional affiliations. 